\title{
Swimming-induced exercise promotes hypertrophy and vascularization of fast skeletal muscle fibres and activation of myogenic and angiogenic transcriptional programs in adult zebrafish
}

\author{
Arjan P Palstra ${ }^{1,2,3+}$, Mireia Rovira ${ }^{1,2 \dagger}$, David Rizo-Roca ${ }^{1}$, Joan Ramon Torrella' ${ }^{1}$, Herman P Spaink ${ }^{4}$ \\ and Josep V Planas ${ }^{1,2^{*}}$
}

\begin{abstract}
Background: The adult skeletal muscle is a plastic tissue with a remarkable ability to adapt to different levels of activity by altering its excitability, its contractile and metabolic phenotype and its mass. We previously reported on the potential of adult zebrafish as a tractable experimental model for exercise physiology, established its optimal swimming speed and showed that swimming-induced contractile activity potentiated somatic growth. Given that the underlying exercise-induced transcriptional mechanisms regulating muscle mass in vertebrates are not fully understood, here we investigated the cellular and molecular adaptive mechanisms taking place in fast skeletal muscle of adult zebrafish in response to swimming.

Results: Fish were trained at low swimming speed $(0.1 \mathrm{~m} / \mathrm{s}$; non-exercised) or at their optimal swimming speed $\left(0.4 \mathrm{~m} / \mathrm{s}\right.$; exercised). A significant increase in fibre cross-sectional area $\left(1.290 \pm 88 \mathrm{vs} .1 .665 \pm 106 \mu^{2}\right)$ and vascularization ( $298 \pm 23$ vs. $458 \pm 38$ capillaries $/ \mathrm{mm}^{2}$ ) was found in exercised over non-exercised fish. Gene expression profiling by microarray analysis evidenced the activation of a series of complex transcriptional networks of extracellular and intracellular signaling molecules and pathways involved in the regulation of muscle mass (e.g. IGF-1/PI3K/mTOR, BMP, MSTN), myogenesis and satellite cell activation (e.g. PAX3, FGF, Notch, Wnt, MEF2, Hh, EphrinB2) and angiogenesis (e.g. VEGF, HIF, Notch, EphrinB2, KLF2), some of which had not been previously associated with exercise-induced contractile activity.

Conclusions: The results from the present study show that exercise-induced contractile activity in adult zebrafish promotes a coordinated adaptive response in fast muscle that leads to increased muscle mass by hypertrophy and increased vascularization by angiogenesis. We propose that these phenotypic adaptations are the result of extensive transcriptional changes induced by exercise. Analysis of the transcriptional networks that are activated in response to exercise in the adult zebrafish fast muscle resulted in the identification of key signaling pathways and factors for the regulation of skeletal muscle mass, myogenesis and angiogenesis that have been remarkably conserved during evolution from fish to mammals. These results further support the validity of the adult zebrafish as an exercise model to decipher the complex molecular and cellular mechanisms governing skeletal muscle mass and function in vertebrates.
\end{abstract}

Keywords: Exercise, Swimming, Growth, Muscle, Transcriptome, Zebrafish

\footnotetext{
* Correspondence: jplanas@ub.edu

${ }^{\dagger}$ Equal contributors

'Departament de Fisiologia i Immunologia, Facultat de Biologia, Universitat

de Barcelona, Barcelona, Spain

${ }^{2}$ Institut de Biomedicina de la Universitat de Barcelona (IBUB), Barcelona,

Spain

Full list of author information is available at the end of the article
}

() Biomed Central (c) 2014 Palstra et al.; licensee BioMed Central. This is an Open Access article distributed under the terms of the Creative Commons Attribution License (http://creativecommons.org/licenses/by/4.0), which permits unrestricted use, distribution, and reproduction in any medium, provided the original work is properly credited. The Creative Commons Public Domain Dedication waiver (http://creativecommons.org/publicdomain/zero/1.0/) applies to the data made available in this article, unless otherwise stated. 


\section{Background}

In all animals, skeletal muscle has evolved to play a fundamental role in locomotion and energy metabolism. The adult skeletal muscle is a post-mitotic tissue with unique plasticity, that is, it has an extraordinary ability to adjust to changes in its physiological environment by altering its excitability, its contractile and metabolic phenotype and its mass. Importantly, skeletal muscle usage is able to exert profound changes in its phenotype. The induction of contractile activity by exercise represents a physiological stimulus that elicits important adaptive responses in skeletal muscle either directly by mechanical strain or indirectly through its ability to increase intracellular calcium levels in response to neural stimulation [1-3]. These adaptive responses, that ultimately serve to increase fitness, are governed by genetic programs involving complex transcriptional responses that depend on the activity of transcription factors and chromatin modifying enzymes $[4,5]$ and are not fully understood, even in mammals. Due to the known beneficial effects of exercise-induced skeletal muscle activity for preventing cardiovascular (e.g. coronary heart disease, hypertension), metabolic (e.g. type 2 diabetes mellitus, obesity) and age-related (e.g. sarcopenia) conditions $[6,7]$ in humans, knowledge on the pathways that participate in the adaptation of skeletal muscle to exerciseinduced activity is of crucial importance for understanding the basic mechanisms involved in this process. This may also be important for assessing possible modulatory effects of exercise on muscle regeneration and for identifying potential pharmaceutical targets useful for the treatment of muscle disorders.

After two decades as a research model, the zebrafish (Danio rerio) has made important contributions to our current knowledge on skeletal muscle developmental biology $[8,9]$ and the pathological basis of neuromuscular disorders, such as muscular dystrophy and myopathies $[10,11]$. This has been possible because the zebrafish skeletal muscle has many molecular features (i.e. a conserved transcriptional network regulating myogenesis), as well as histological and ultrastructural features, that are very similar to those in the mammalian muscle $[12,13]$. Furthermore, the zebrafish has anatomically separated fast- and slow-twitch fibres as a result of distinct ontogenic programs making this an interesting model to investigate fibre type specification [9] and fibre growth $[14,15]$. Therefore, the zebrafish, due its tractability and the ease of genetic manipulation coupled with the vast genetic and genomic tools available, has tremendous potential to contribute importantly to our knowledge on skeletal muscle function and, specifically, on the mechanisms responsible for the regulation of adult muscle mass in vertebrates, including humans. However, most of the current knowledge on the regulation of skeletal muscle mass in zebrafish is derived from studies on the effects of muscle inactivity or injury and on genetic models of human muscle disorders $[10,14,16]$ and not based on models of increased skeletal muscle activity, such as induced by exercise. In order to begin to elucidate the effects of exercise-induced contractile activity on skeletal muscle physiology in adult zebrafish and to contribute to its establishment as an exercise model species in fish and biomedical research, we recently studied the swimming economy in adult zebrafish and established its optimal swimming speed (i.e. the swimming speed at which the cost of transport is lowest and the energetic efficiency is highest) [17]. By applying these aerobic exercise conditions in a swimming training protocol for 20 days, a significant exercise-induced growth was demonstrated for the first time in adult zebrafish that was associated with the regulated expression of growth marker genes in fast muscle [17]. Based on the results from that study, we put forward the notion that zebrafish can be used as an exercise model for studying muscle growth. Therefore, the present study aimed to describe the cellular and molecular adaptive response of fast skeletal muscle to swimming-induced exercise in adult zebrafish and further validate the zebrafish as a useful animal model for investigating the effects of exercise on skeletal muscle physiology in vertebrates.

In the present study, we report on the effects of exercise training on the cellular and molecular characteristics of fast muscle in adult zebrafish. Our results indicate that exercise-induced contractile activity in adult zebrafish promotes a coordinated adaptive response in fast muscle that leads to increased muscle mass by hypertrophy and increased vascularization by angiogenesis. These phenotypic changes are likely the result of the transcriptional activation of a series of complex networks of extracellular and intracellular signaling molecules and pathways involved in the regulation of muscle mass, myogenesis and angiogenesis in adult zebrafish, some not previously associated with exercise-induced contractile activity. Moreover, the present study reinforces the notion that zebrafish is a valid and promising animal model to promote our understanding of the complex mechanisms responsible for the regulation of adult skeletal muscle mass by exercise.

\section{Results}

Exercise training promotes changes in fibre morphometry and capillarization in fast muscle of adult zebrafish

Morphometrical assessment of fast muscle in exercised and non-exercised adult zebrafish was performed to evaluate the effects of exercise training on skeletal muscle cellular characteristics (Figure 1A-D). Exercised zebrafish showed a significant $(\mathrm{P}<0.05)$ increase $(29 \%)$ in fibre cross-sectional area (FCSA) (Figure 1E). Furthermore, exercised zebrafish also showed a significant $(\mathrm{P}<0.05)$ increase in fibre perimeter $(12 \%)$ (Figure 1F) 

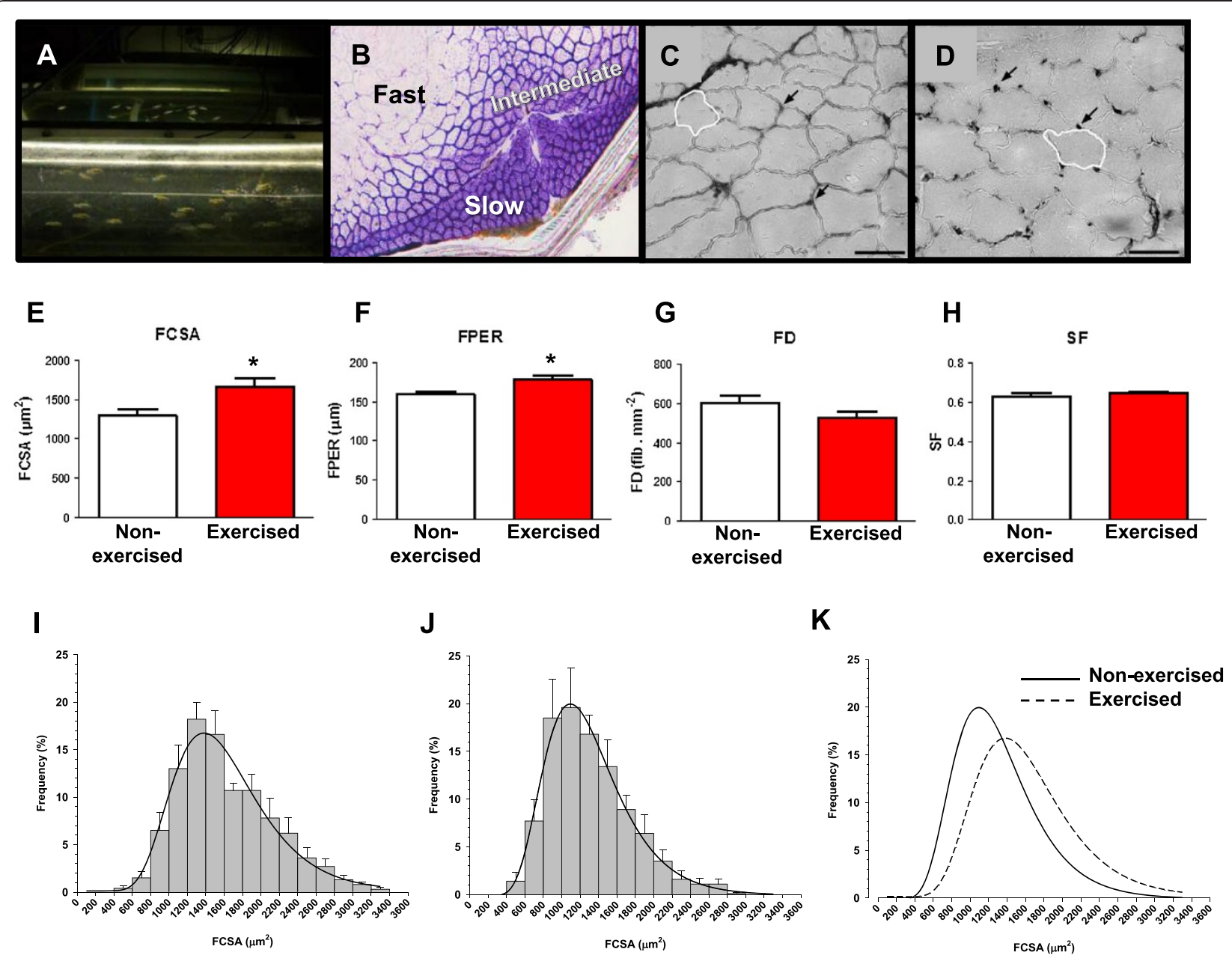

Figure 1 Morphometrical fibre parameters in fast muscle of exercised and non-exercised adult zebrafish. A: Image of the swim tunnels used for exercise training. Front tunnel: exercised zebrafish; back tunnel: non-exercised zebrafish. B-D: Images of zebrafish cross-sectional white muscle. Images correspond to representative serial transverse secions stained (B) for succinate dehydrogenase for the identification of fast, intermediate (pink) and slow muscle fibres; (C and D) for ATPase for capillary demonstration (arrows) and FCSA and FPER measures (white drawing) from a non-exercised (C) and an exercised (D) adult zebrafish. Bar represents $50 \mu \mathrm{m}$. Morphometric fibre parameters measured in non-exercised and exercised zebrafish were: $F C S A$, fibre cross-sectional area $\left(\mu \mathrm{m}^{2}\right) \mathbf{( E )}$; $F P E R$, fibre perimeter $(\mu \mathrm{m})(\mathbf{F}) ; F D$, fibre density (fibres $\left./ \mathrm{mm}^{2}\right)(\mathbf{G})$; $S F$, shape factor (H). Statistical significance values between non-exercised and exercised zebrafish: $* P<0.05$. Values are mean \pm SEM from a sample size of $n=8$ for each condition. I-J: Fibre cross-sectional area histograms from fast muscle of exercised (I) and non-exercised (J) adult zebrafish. In K, the two overlapped curves are shown. Muscle fibre areas were grouped in intervals of $200 \mu^{2}$ and the data correspond to mean \pm SEM frequency of six animals. Curves represent a log-normal regression of four parameters. Regression parameters are shown in Additional file 1. See Methods for details.

and a non-significant decrease in fibre density (Figure 1G) in fast muscle without changes in the shape of the fibres, as indicated by the absence of differences in fibre circularity (shape factor) between exercised and non-exercised zebrafish (Figure $1 \mathrm{H})$. Fast muscle fibre frequency distribution analyses in non-exercised and exercised zebrafish evidenced that log-normal regression curves were centered around higher FCSA values in exercised (approximately $1.400 \mu^{2}$ ) (Figure 1I) over non-exercised zebrafish (approximately $1.100 \mu \mathrm{m}^{2}$ ) (Figure $1 \mathrm{~J}$ ), as also deduced by the significant $(\mathrm{P}<0.0001)$ shift to the right of the regression curve of exercised zebrafish relative to that of non-exercised zebrafish (Figure 1K; Additional file 1: Table S1). When the mean percentages of muscle fibres were grouped into three major intervals of FCSA and quantified, exercised zebrafish presented significantly lower percentages of small fibres (FCSA $<1.200 \mu \mathrm{m}^{2}$ ) but significantly higher percentages of medium (with sizes between $1.200 \mu \mathrm{m}^{2}$ and $2.400 \mu \mathrm{m}^{2}$ ) and large fibres (FCSA $>2.400$ $\mu \mathrm{m}^{2}$ ) than non-exercised zebrafish (Additional file 1: Table S1). Therefore, these observations clearly indicate that fibre size was significantly increased in exercised zebrafish and, consequently, that exercise training caused hypertrophy of fast muscle fibres in adult zebrafish. 
Exercise training also induced vascularization of the fast muscle in zebrafish, as assessed by histochemical quantification of capillaries (Figure 1C,D). The total capillary density increased by $54 \%(\mathrm{P}<0.01)$ in fast muscle of exercised relative to non-exercised zebrafish (Figure 2A). Importantly, exercise training caused a significant $(\mathrm{P}<0.001)$ increase in the number of capillaries in contact with each fibre (98\%) (Figure 2B) as well as a significantly greater number of capillaries per fibre area (52\%) (Figure 2C) and per fibre perimeter (76\%) (Figure 2D) in fast muscle of adult zebrafish. The capillary-to-fibre ratio (CD/FD) increased by $74 \%$ $(\mathrm{P}<0.001)$ in exercised zebrafish (Figure 2E). However, maximal diffusion distance between the capillary and the centre of the fibre was modestly but significantly $(\mathrm{P}<0.05)$ increased $(15 \%)$ in the fast muscle of exercised zebrafish (Figure 2F), likely as a result of a greater fibre size.

\section{Exercise training induces profound transcriptomic changes in fast muscle of adult zebrafish}

In order to gain insight into the molecular basis of the increase in fast muscle fibre hypertrophy and vascularization in exercised adult zebrafish, we evaluated the transcriptomic response of fast muscle to swimming-induced exercise by microarray analysis. Gene expression profiling of the zebrafish fast muscle evidenced important transcriptomic changes, with 1.625 genes down-regulated and 2.851 genes up-regulated in response to exercise training. Initial classification of differentially expressed genes by Gene Ontology categories using DAVID revealed a significant $(\mathrm{p}<0.05)$ enrichment in functional categories related
A

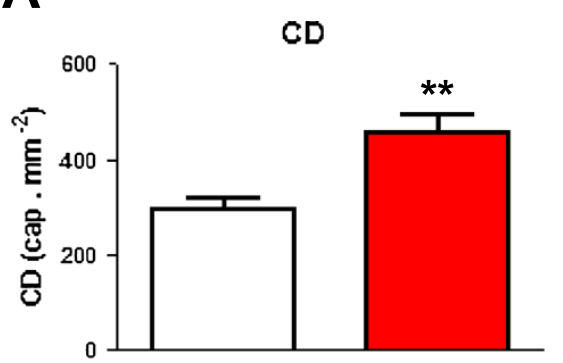

C

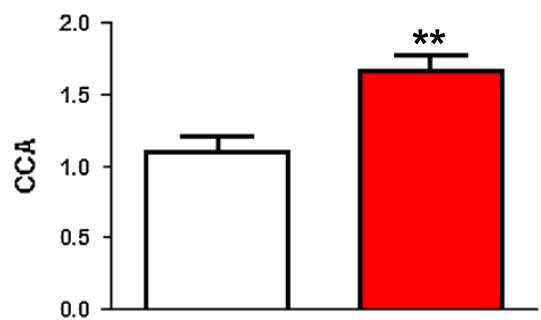

E

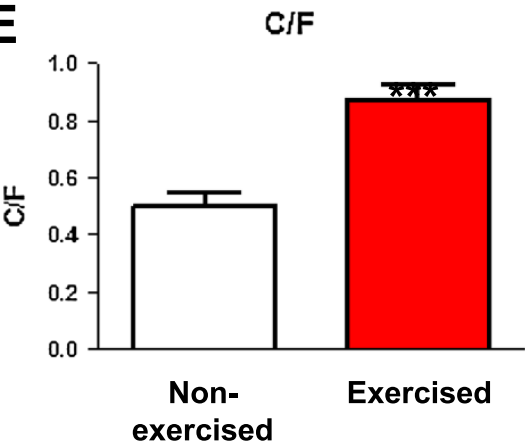

B

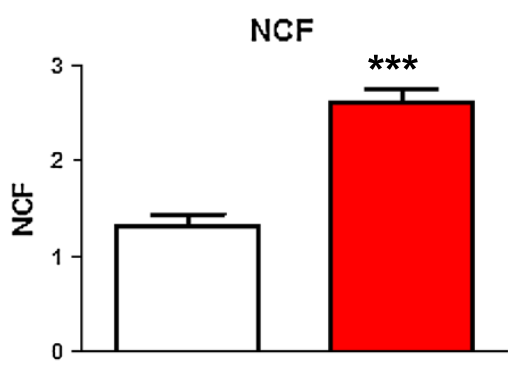

D

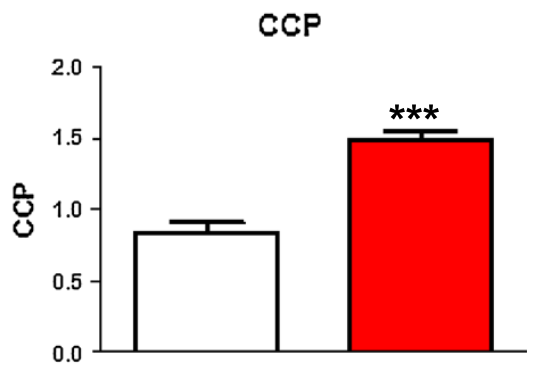

$\mathbf{F}$

MDD

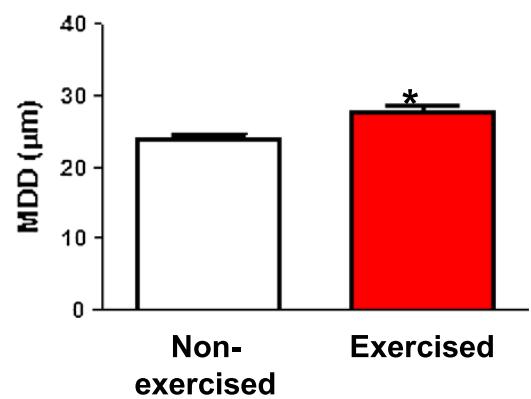

Figure 2 Morphometrical capillarity parameters in fast muscle of exercised and non-exercised adult zebrafish. Parameters measured were: $C D$, capillary density (capillaries $/ \mathrm{mm}^{2}$ ) $(\mathbf{A})$; NCF, number of capillaries in contact with each fibre (B); CCA, relationship between NCF and the FCSA (NCF $10^{3} /$ FCSA) (C); CCP, relationship between NCF and the FPER (NCF $\left.10^{2} / F P E R\right)(D) ; C / F$, capillary-to-fibre ratio (CD/FD) (E) and MDD, maximal diffusion distance between the capillary and the centre of the fibre $\mathbf{( F )}$. Statistical significance values between non-exercised and exercised zebrafish: ${ }^{*} \mathrm{P}<0.05,{ }^{*} \mathrm{P}<0.01,{ }^{* * *} \mathrm{P}<0.001$. Values are mean \pm SEM from a sample size of $\mathrm{n}=8$ for each condition. 
to muscle development and differentiation, sarcomeric contractile elements, cell cycle and apoptosis, protein, carbohydrate and lipid metabolism, oxidative phosphorylation and blood vessel development (Table 1). Importantly, exercise training modulated the expression of genes involved in a wide variety of processes that are responsible for the functional contractile activation of skeletal muscle fibres: activation of neuromuscular communication (e.g. ache, chrm2, scn $4 b$ ), translation of nerve-evoked electrical activity into an intracellular $\mathrm{Ca}^{2+}$

Table 1 Functional annotation analysis based on GO terms in zebrafish fast muscle in response to swimming (DAVID)

\begin{tabular}{|c|c|c|c|}
\hline GO Term & & Count & P-value \\
\hline GO:0014706 & Striated muscle tissue development & 41 & 0,0014644 \\
\hline GO:0051146 & Striated muscle cell differentiation & 31 & 0,0042799 \\
\hline GO:0030239 & Myofibril assembly & 10 & 0,0227268 \\
\hline GO:0031032 & Actomyosin structure organization & 14 & 0,0028624 \\
\hline GO:0032956 & Regulation of actin cytoskeleton organization & 28 & 0,0318891 \\
\hline GO:0040007 & Growth & 65 & $1,72 \mathrm{E}-05$ \\
\hline GO:0045926 & Negative regulation of growth & 36 & 0,0074826 \\
\hline GO:0000278 & Mitotic cell cycle & 124 & $8,01 \mathrm{E}-08$ \\
\hline GO:0051726 & Regulation of cell cycle & 92 & 0,0053137 \\
\hline GO:0006915 & Apoptosis & 161 & 0,0014411 \\
\hline GO:0043065 & Positive regulation of apoptosis & 110 & 0,0295542 \\
\hline GO:0006457 & Protein folding & 60 & 1,67E-04 \\
\hline GO:0030162 & Regulation of proteolysis & 19 & 0,0245616 \\
\hline GO:0006468 & Protein amino acid phosphorylation & 173 & 0,0038954 \\
\hline GO:0006511 & Ubiquitin-dependent protein catabolic process & 84 & $2,55 \mathrm{E}-06$ \\
\hline GO:0006979 & Response to oxidative stress & 46 & 0,0414755 \\
\hline GO:0080135 & Regulation of cellular response to stress & 31 & 0,0418028 \\
\hline GO:0045454 & Cell redox homeostasis & 21 & 0,0378637 \\
\hline GO:0015980 & Energy derivation by oxidation of organic compounds & 51 & $1,68 \mathrm{E}-04$ \\
\hline GO:0022900 & Electron transport chain & 41 & $5,67 \mathrm{E}-04$ \\
\hline GO:0006754 & ATP biosynthetic process & 32 & 0,0025614 \\
\hline GO:0006119 & Oxidative phosphorylation & 44 & $5,45 \mathrm{E}-07$ \\
\hline GO:0044262 & Cellular carbohydrate metabolic process & 111 & $6,80 \mathrm{E}-04$ \\
\hline GO:0006096 & Glycolysis & 21 & $9,35 \mathrm{E}-04$ \\
\hline GO:0044255 & Cellular lipid metabolic process & 157 & 4,84E-06 \\
\hline GO:0006635 & Fatty acid beta-oxidation & 15 & 7,99E-04 \\
\hline GO:0006631 & Fatty acid metabolic process & 75 & 2,13E-07 \\
\hline GO:0006633 & Fatty acid biosynthetic process & 30 & 0,0013958 \\
\hline GO:0006520 & Cellular amino acid metabolic process & 71 & $5,16 \mathrm{E}-04$ \\
\hline GO:0042180 & Cellular ketone metabolic process & 190 & $1,95 \mathrm{E}-11$ \\
\hline GO:0001568 & Blood vessel development & 78 & $1,68 \mathrm{E}-04$ \\
\hline GO:0048514 & Blood vessel morphogenesis & 68 & $3,13 \mathrm{E}-04$ \\
\hline GO:0001570 & Vasculogenesis & 16 & 0,0194009 \\
\hline GO:0045449 & Regulation of transcription & 593 & 0,0425425 \\
\hline GO:0043408 & Regulation of MAPKKK cascade & 35 & 0,011466 \\
\hline GO:0051101 & Regulation of DNA binding & 42 & 0,0010788 \\
\hline GO:0007243 & Protein kinase cascade & 95 & 0,0387284 \\
\hline GO:0030509 & BMP signaling pathway & 17 & 0,0170619 \\
\hline GO:0016055 & Wnt receptor signaling pathway & 53 & $2,84 \mathrm{E}-06$ \\
\hline
\end{tabular}


signal (i.e. excitation-contraction coupling) (e.g. atp2a1, calm1, casq1, pvalb, ppp3ca, ryr1), sarcomere contraction (e.g. actn4, actb, actc1, capzb, mybph, myh11, myl2, myl9, mylpf, tpm1, tnni2, tnnt3, ttn), cytoskeletal transmission of sarcomeric contractile force to the sarcolemma (e.g. ank2, dag1, des, dmd, dtnbp1, flnc, itga2b, itgb4, lmna, myoz1, myoz2, sntb1, sptbn, vim) and force transmission and muscle structure maintenance by the extracellular matrix (e.g. col1a1, colsa2, col16a1, lama1, lamc3, loxl2, loxl3, $s d c b p$, tnc) (Table 2). Furthermore, exercise training also altered the expression of fast muscle genes involved in the control of muscle growth and development, such as growth factors (e.g. egfr, fgf13, fgf18, fgf20, fgfr1, fgfr2, fst, igflr, igfbp1, igfbp3, igfbp7, igf2, mstn, ngf, tgfb1, tgfb2), extracellular signaling molecules (e.g. bmp1, bmp4, bmpr1a, bmpr1b, ihh, nog, shh, wnt7a, wnt10a), components of intracellular signaling pathways (e.g. esrra, esrrb, esrrg, foxa1, foxo3, irs1, irs2, mapk1, mapk8, mapk13, mapk14pik3c2b, smad6) and transcriptional regulators of myogenesis (e.g. hdac4, hadc6, id1, id3, mef2a, mef2ca, mef $2 d$, pax3) (Table 2).

Consistent with the increased vascularization of fast muscle by exercise training, the expression of a number of genes involved in angiogenesis was altered in fast muscle, including angiopoietins (e.g. angpt2, angptl2, angptl3), members of the ephrin family and receptors (e.g. efna2, efna3, efnb2, efnb3, epha4, epha7, ephb4), members of the notch family (e.g. dll1, jag1, jag2, notch1, notch2), hypoxia-inducible factors (e.g. hif1an, hif3a), gata1 and nrp1 (Table 3). Among genes involved in metabolism with altered mRNA expression levels in fast muscle of exercised zebrafish were genes responsible for the metabolic provision of ATP in skeletal muscle such as pdha1, members of the ATP-phosphagen system (e.g. $a k 1, a k 2, a 3, c k m, c k m t 2)$, and multiple components of the mitochondrial electron transport chain (e.g. ndufa, cox, atp5) and the tricarboxylic acid (TCA) cycle (e.g. fh, idh3b, idh3g, mdh1, mdh2, ogdh, sdha) (Table 3). Other differentially expressed genes included genes known to participate in energy metabolism (e.g. adipor $2, m b$, prkaa1, prkab1, prkag1, ppara, ppard, ucp2 and ucp3). Moreover, genes involved in the metabolic utilization of energy substrates as fuel, namely lipids (e.g. cpt2, capt1a, fabp3, lpl, mcat, slc27a2) and carbohydrates (e.g. aldoa, aldoc, eno1, gapdh, g6pc, gpi, hk2, pfkm, pgk1, $p k m$ ), also showed altered expression in fast muscle of exercised zebrafish. Importantly, exercise training altered the expression of genes involved in protein synthesis and degradation in fast muscle (e.g. eifte, eiftebp1, fbxo32, foxo3, pdk1, pdk2, rps6ka1, trim63). Finally, exercise training caused alterations in the expression of immunerelated genes (e.g. il11ra, il12b, il13ra2, il17d, ill7dr, il20, il20ra, irf3, mif, mst1 and traf6) in fast muscle of adult zebrafish (Table 3 ).
We further analyzed the transcriptomic effects of exercise training on the fast muscle of adult zebrafish by mining the Ingenuity Knowledge Base for biological functions, pathways and networks. Among the biological functions that showed highly significant $(\mathrm{P}<0.00001)$ changes in fast muscle in response to exercise were muscle development, myogenesis, angiogenesis, cell cycle progression, mitosis, cytoskeleton organization, lipid oxidation, lipid synthesis and organismal growth (Additional file 2: Table S2), with 143, 59, 230, 408, 172, 424, 81, 240 and 201 differentially expressed genes, respectively. The lists of differentialy expressed genes involved in muscle development, myogenesis, angiogenesis and cell proliferation are shown in Additional files 3, 4, 5 and 6: Tables S3-S6. Canonical pathway analysis identified 22 pathways that were significantly $(\mathrm{P}<0.05)$ overrepresented in fast muscle of adult exercised zebrafish (Table 4). Regulated canonical signaling pathways associated with skeletal muscle contractile activity included the calcium, integrin, actin cytoskeleton, FGF, wnt $/ \beta$-catenin and AMPK signaling pathways. Moreover, the IGF-1, insulin receptor, PI3K/AKT and mTOR signaling pathways were also significantly regulated in fast muscle, in accordance with the observed hypertrophy in fast muscle of exercised zebrafish. Interestingly, the canonical TGF $\beta$ signaling pathway was also significantly altered by exercise in fast muscle. The metabolic effects of exercise training in the zebrafish fast muscle were exemplified by the significant regulation of the protein ubiquitination pathway, glycolysis and fatty acid $\beta$-oxidation. Furthermore, exercise training also caused a significant over-representation of signaling pathways involved in angiogenesis (e.g. ephrin B, VEGF, hypoxia, PDGF, HIF1 $\alpha$, Notch and angiopoietin signaling pathways) in the zebrafish fast muscle (Table 4). The genes that are differentially regulated by exercise training that correspond to each of the over-represented canonical pathways are listed in Additional file 7: Table S7.

Analysis of gene networks corresponding to muscle development and angiogenesis by IPA allowed us to establish connectivity maps for these two processes (Figures 3 and 4). The connectivity map of regulated genes involved in muscle development illustrates nodes around transcription factors and nuclear genes such as ccna2, crebbp, ep300, hdac1, kfl2, mef2c, mef2d, pax3, rela, smad7, srf and tp63, that are integrated with key sarcomeric and cytoskeletal elements and key signaling molecules and transducers of extracellular signals involved in the regulation of this process (e.g. bmp4, dll1, fst, igf2, ihh, jag1, $m s t n$, shh, tgfb1, wnt1, wnt2) (Figure 3). Regulated genes involved in angiogenesis show a connectivity map with nodes around the nuclear factors ctnnb1, crebbp, foxc1, klf2, runx2, tfap $2 a, t p 53$ and sirt1 that are clearly integrated with extracellular signals (e.g. angpt2, bmp4, edn1, 
Table 2 Selected differentially expressed genes in fast muscle of exercised zebrafish that participate in the contractile activation of skeletal muscle fibers

\begin{tabular}{|c|c|c|c|c|c|}
\hline \multicolumn{2}{|c|}{ Gene name gene description } & \multirow{3}{*}{\begin{tabular}{|c|} 
FC \\
4.11
\end{tabular}} & \multicolumn{2}{|c|}{ Gene name gene description } & \multirow{3}{*}{$\begin{array}{r}\text { FC } \\
8.94\end{array}$} \\
\hline \multicolumn{2}{|c|}{ Muscle contraction } & & \multicolumn{2}{|c|}{ Muscle growth and development } & \\
\hline capn8 & Calpain 8 & & fgf20 & Fibroblast growth factor 20 & \\
\hline $\operatorname{actn} 4$ & Actinin, alpha 4 & 3.99 & hdac6 & Histone deacetylase 6 & 6.42 \\
\hline myh11 & Myosin, heavy chain 11 , smooth muscle & 3.63 & fgf18 & Fibroblast growth factor 18 & 6.30 \\
\hline camk2n2 & $\mathrm{Ca} / \mathrm{calmodulin-dependent} \mathrm{protein} \mathrm{kinase} \mathrm{II} \mathrm{inhibitor} 2$ & 3.38 & wnt10a & Wingless-type MMTV integration site, $10 \mathrm{~A}$ & 6.25 \\
\hline pvalb & Parvalbumin & 3.24 & pax3 & Paired box 3 & 6.21 \\
\hline thni2 & Troponin I type 2 (skeletal, fast) & 3.12 & $\operatorname{tgfb2}$ & Transforming growth factor, beta 2 & 5.35 \\
\hline capn3 & Calpain 3, (p94) & 3.08 & nog & Noggin & 4.90 \\
\hline nfatc4 & Nuclear factor of activated T-cells, calcineurin-dep. 4 & 3.08 & esrra & Estrogen-related receptor alpha & 4.73 \\
\hline capn2 & Calpain 2, (m/II) large subunit & 2.92 & wnt7a & Wingless-type MMTV integration site, 7A & 4.60 \\
\hline tmod4 & Tropomodulin 4 (muscle) & 2.79 & mstn & Myostatin & 4.41 \\
\hline nfatcl & Nuclear factor of activated T-cells, , calcineurin-dep. 1 & 2.76 & foxal & Forkhead box A1 & 4.22 \\
\hline capzb & Capping protein (actin filament) muscle Z-line, beta & 2.75 & fgfr2 & Fibroblast growth factor receptor 2 & 4.05 \\
\hline casq1 & Calsequestrin 1 (fast-twitch, skeletal muscle) & 2.68 & shh & Sonic hedgehog & 3.78 \\
\hline myl2 & Myosin, light chain 2, regulatory, cardiac, slow & 2.64 & $f z d 2$ & Frizzled family receptor 2 & 3.08 \\
\hline ppp3cc & Protein phosphatase 3, catalytic subunit, gamma & 2.61 & pik3c2b & Phosphatidylinositol-4-p- 3-kinase c2b & 3.06 \\
\hline capn5 & Calpain 5 & 2.60 & fgfi3 & Fibroblast growth factor 13 & 3.01 \\
\hline $\operatorname{ttn}$ & Titin & 2.58 & mapk1 & Mitogen-activated protein kinase 1 & 3.00 \\
\hline рррзса & Protein phosphatase 3, catalytic subunit, alpha isozyme & 2.52 & $f z d 10$ & Frizzled family receptor 10 & 2.94 \\
\hline mylpf & Myosin light chain, phosphorylatable, fast skel. muscle & 2.26 & ihh & Indian hedgehog & 2.91 \\
\hline mybph & Myosin binding protein $\mathrm{H}$ & 2.17 & $f z d 8$ & Frizzled family receptor 8 & 2.87 \\
\hline capn10 & Calpain 10 & 2.15 & esrrb & Estrogen-related receptor beta & 2.61 \\
\hline cacnals & Calcium channel, voltage-dependent, L type, alpha is & 2.11 & bmpria & Bone morphogenetic protein receptor, IA & 2.58 \\
\hline camk2a & Calcium/calmodulin-dependent protein kinase II alpha & 1.98 & $n g f$ & Nerve growth factor (beta polypeptide) & 2.55 \\
\hline camk2d & Calcium/calmodulin-dependent protein kinase II delta & 1.97 & igfir & Insulin-like growth factor 1 receptor & 2.53 \\
\hline nfatc3 & Nuclear factor of activated T-cells, calcineurin-dep. 3 & 1.92 & bmp1 & Bone morphogenetic protein 1 & 2.46 \\
\hline acta2 & Actin, alpha 2, smooth muscle, aorta & 1.92 & $d v / 1$ & Dishevelled, dsh homolog 1 (Drosophila) & 2.43 \\
\hline mylk & Myosin light chain kinase & 1.87 & smad2 & SMAD family member 2 & 2.40 \\
\hline tpm4 & Tropomyosin 4 & 1.78 & bmp4 & Bone morphogenetic protein 4 & 2.38 \\
\hline myl9 & Myosin, light chain 9, regulatory & 1.77 & igfbp7 & Insulin-like growth factor binding protein 7 & 2.36 \\
\hline ryr1 & Ryanodine receptor 1 (skeletal) & 1.77 & esrrg & Estrogen-related receptor gamma & 2.36 \\
\hline tpm 1 & Tropomyosin 1 (alpha) & 1.71 & bmprib & Bone morphogenetic protein receptor, IB & 2.27 \\
\hline atp2a1 & ATPase, Ca transporting, cardiac muscle, fast twitch 1 & 1.70 & erbb2 & v-erb-b2 erythroblastic leukemia. 2 & 2.27 \\
\hline actc1 & Actin, alpha, cardiac muscle 1 & 1.68 & mapk13 & Mitogen-activated protein kinase 13 & 2.23 \\
\hline cacngl 1 & Calcium channel, voltage-dependent, gamma subunit 1 & 1.61 & fst & Follistatin & 2.17 \\
\hline myl12b & Myosin, light chain $12 \mathrm{~B}$, regulatory & -1.59 & mapk8 & Mitogen-activated protein kinase 8 & 2.12 \\
\hline s100a4 & S100 calcium binding protein A4 & -1.63 & smad6 & SMAD family member 6 & 2.06 \\
\hline calm1 & Calmodulin 1 (phosphorylase kinase, delta) & -2.04 & fgfr1 & Fibroblast growth factor receptor 1 & 1.96 \\
\hline $\operatorname{actg} 2$ & Actin, gamma 2, smooth muscle, enteric & -3.87 & irs2 & Insulin receptor substrate 2 & 1.91 \\
\hline thnt3 & Troponin T type 3 (skeletal, fast) & -7.01 & runx2 & Runt-related transcription factor 2 & 1.90 \\
\hline \multicolumn{2}{|c|}{ Cytoskeleton } & & igfbp 1 & Insulin-like growth factor binding protein 1 & 1,89 \\
\hline ank2 & Ankyrin 2, neuronal & 11.01 & irs1 & Insulin receptor substrate 1 & 1.78 \\
\hline plec & Plectin & 3.31 & $a c v r 2 b$ & Activin $A$ receptor, type $\| B$ & 1.74 \\
\hline
\end{tabular}


Table 2 Selected differentially expressed genes in fast muscle of exercised zebrafish that participate in the contractile activation of skeletal muscle fibers (Continued)

\begin{tabular}{|c|c|c|c|c|c|}
\hline myoz1 & Myozenin 1 & 2.41 & $\operatorname{tgfb} 1$ & Transforming growth factor, beta 1 & 1.71 \\
\hline myoz2 & Myozenin 2 & 2.28 & mef2d & Myocyte enhancer factor $2 \mathrm{D}$ & 1.71 \\
\hline $\operatorname{dag} 1$ & Dystroglycan 1 (dystrophin-associated glycoprotein 1) & 2.26 & hdac4 & Histone deacetylase 4 & 1.71 \\
\hline itgb4 & Integrin, beta 4 & 1.99 & igfbp3 & Insulin-like growth factor binding protein 3 & 1.66 \\
\hline$i \operatorname{tg} a 2 b$ & Integrin, alpha $2 b$ & 1.95 & mef2a & Myocyte enhancer factor 2A & 1.66 \\
\hline$d m d$ & Dystrophin & 1.94 & igf2 & Insulin-like growth factor 2 & 1.61 \\
\hline filip1 & Filamin A interacting protein 1 & 1.88 & pten & Phosphatase and tensin homolog & -1.54 \\
\hline sntb1 & Syntrophin, beta 1 (dystrophin-associated protein A1) & 1.78 & mef2c & Myocyte enhancer factor $2 \mathrm{C}$ & -1.59 \\
\hline vim & Vimentin & 1.61 & egfr & Epidermal growth factor receptor & -2.13 \\
\hline Imna & Lamin $\mathrm{A} / \mathrm{C}$ & -1.51 & $i d 3$ & Inhibitor of DNA binding 3 & -2.39 \\
\hline$d$ tnbp 1 & Dystrobrevin binding protein 1 & -1.76 & srf & Serum response factor & -2.68 \\
\hline flnc & Filamin C, gamma & -1.88 & mapk14 & Mitogen-activated protein kinase 14 & -2.78 \\
\hline \multicolumn{3}{|c|}{ Neuromuscular junction } & \multicolumn{3}{|c|}{ Extracelular matrix } \\
\hline ache & Acetylcholinesterase & 8.86 & colsa2 & Collagen, type VIII, alpha 2 & 12.06 \\
\hline vamp1 & Vesicle-associated membrane prot. 1 (synaptobrevin1) & 3.78 & lamc3 & Laminin, gamma 3 & 10.06 \\
\hline chrm2 & Cholinergic receptor, muscarinic 2 & 3.65 & col16al & Collagen, type XVI, alpha 1 & 6.17 \\
\hline snap25 & Synaptosomal-associated protein, $25 \mathrm{kDa}$ & 3.08 & colla2 & Collagen, type I, alpha 2 & 3.12 \\
\hline $\operatorname{scn} 4 b$ & Sodium channel, voltage-gated, type IV, beta subunit & 3.01 & bgn & Biglycan & 2.98 \\
\hline syn2 & Synapsin II & 2.70 & $10 \times 12$ & Lysyl oxidase-like 2 & 2.92 \\
\hline syt1 & Synaptotagmin I & 2.22 & mmp14 & Matrix metallopeptidase 14 & 2.69 \\
\hline rims2 & Regulating synaptic membrane exocytosis 2 & 1.93 & the & Tenascin C & 2.53 \\
\hline scnml & Sodium channel modifier 1 & 1.65 & mmp10 & Matrix metallopeptidase 10 (stromelysin 2) & 2.06 \\
\hline syncrip & Synaptotagmin binding, cytoplasmic RNA interact. pro. & -1.54 & sdcbp & Syndecan binding protein (syntenin) & -2.26 \\
\hline
\end{tabular}

Data are shown as fold change (FC).

Functional categories are indicated in bold.

$f g f 13$, igf2, jag1, pdgfa, vegfc) transducing their effects primarily through the efnb2, erbb2, fgf, igf1 and notch signaling pathways via molecules such as irs1, mapk1, mapk8, nos 2 and pik3cg among others (Figure 4).

The results of microarray analysis were validated by qPCR for 7 differentially expressed genes in fast muscle: 4 down-regulated (fabp 7, tuba1b, psme3, psma5) and 3 up-regulated (capns1, fgfrl1, foxal) genes. The genes examined showed a similar pattern of change with the two techniques used, except for capns1 (Additional file 8: Table S8).

\section{Discussion}

\section{Exercise training induces growth of fast muscle fibers in adult zebrafish}

The present study describes the cellular and molecular adaptive mechanisms that are responsible for the plasticity of fast skeletal muscle to exercise-induced contractile activity. Here, we have adopted swimming adult zebrafish as a muscle activity model and have shown, for the first time in adult zebrafish, that exercise training under sustained, aerobic conditions causes hypertrophy of fast muscle fibres. We hypothesize that this may explain, at least in part, the stimulation of muscle growth by swimming in adult zebrafish that we previously reported using the same experimental conditions [17]. Therefore, as in mammals $[4,18]$ and in other fish species [19], exercise promotes growth in adult zebrafish by increasing muscle mass as a result of increased fibre hypertrophy.

Our gene expression analysis of fast muscle of exercised adult zebrafish shows that the increase in fibre hypertrophy is associated with an important regulation of the fast muscle transcriptome. Here, we show for the first time in zebrafish that exercise-stimulated contractile activity in adult fast muscle induced significant and parallel changes in the expression of canonical pathways important for the regulation of protein turnover, namely the anabolic IGF-1/PI3K/Akt/mTOR signaling pathways that promote protein synthesis and the catabolic ubiquitination and atrophy pathways that are responsible for protein degradation [18]. The increase in the expression of genes involved in protein synthesis and in its regulation (e.g. igfr1, irs1, pi3k, pdk1, pdk2, rps6ka1) and the decrease in the expression of the translation inhibitor eif 4 ebp 1 
Table 3 Selected differentially expressed genes in fast muscle of exercised adult zebrafish that participate in angiogenesis, immune-related processess and metabolism

\begin{tabular}{|c|c|c|c|c|c|}
\hline \multicolumn{2}{|c|}{ Gene name gene description } & \multirow[t]{2}{*}{ FC } & \multicolumn{2}{|c|}{ Gene name gene description } & \multirow[t]{2}{*}{ FC } \\
\hline \multicolumn{2}{|c|}{ Angiogenesis } & & \multicolumn{2}{|c|}{ Energy metabolism } & \\
\hline$k \mid f 2$ & Kruppel-like factor 2 (lung) & 8.52 & cpt1a & Carnitine palmitoyltransferase 1A (liver) & 5.23 \\
\hline robo2 & $\begin{array}{l}\text { Roundabout, axon guidance receptor, homolog } \\
2 \text { (Drosophila) }\end{array}$ & 4.30 & $p f k m$ & Phosphofructokinase, muscle & 3.84 \\
\hline angpt2 & Angiopoietin 2 & 3.90 & prkaaq & Protein kinase, AMP-activated, alpha 1 cat. & 3.66 \\
\hline angpt/3 & Angiopoietin-like 3 & 3.47 & elov/4 & ELOVL fatty acid elongase 4 & 3.65 \\
\hline efna3 & Ephrin-A3 & 3.45 & prkag1 & $\begin{array}{l}\text { Protein kinase, AMP-activated, gamma } 1 \\
\text { catalytic subunit }\end{array}$ & 3.50 \\
\hline gatal & $\begin{array}{l}\text { GATA binding protein } 1 \text { (globin transcription } \\
\text { factor 1) }\end{array}$ & 3.00 & acadl & Acyl-CoA dehydrogenase, long chain & 3.20 \\
\hline epha4 & $\mathrm{EPH}$ receptor $\mathrm{A} 4$ & 2.96 & ppard & Peroxisome proliferator-activated receptor $\mathrm{d}$ & 3.19 \\
\hline nrp1 & Neuropilin 1 & 2.89 & aldoa & Aldolase A, fructose-bisphosphate & 3.18 \\
\hline mmp14 & Matrix metallopeptidase 14 (membrane-inserted) & 2.69 & mcat & Malonyl CoA:ACP acyltransferase (mitochondrial) & 3.14 \\
\hline nos1 & Nitric oxide synthase 1 (neuronal) & 2.65 & s/c27a2 & Solute carrier family 27 (fatty acid transporter), member 2 & 3.10 \\
\hline notch1 & Notch 1 & 2.60 & prkab1 & $\begin{array}{l}\text { Protein kinase, AMP-activated, beta } 1 \\
\text { non-catalytic subunit }\end{array}$ & 3.06 \\
\hline sema3f & $\begin{array}{l}\text { Sema domain, immunoglobulin domain (lg), short } \\
\text { basic domain, secreted, (semaphorin) 3F }\end{array}$ & 2.57 & $m b$ & Myoglobin & 2.95 \\
\hline slit3 & Slit homolog 3 (Drosophila) & 2.53 & $\operatorname{cox} 7 \mathrm{C}$ & Cytochrome c oxidase subunit VIIc & 2.85 \\
\hline amot & Angiomotin & 2.34 & ppara & Peroxisome proliferator-activated receptor alpha & 2.77 \\
\hline hey2 & Hairy/enhancer-of-split related with YRPW motif 2 & 2.20 & $t f b 2 m$ & Transcription factor B2, mitochondrial & 2.42 \\
\hline tp63 & Tumor protein p63 & 2.16 & $f b p 1$ & Fructose-1,6-bisphosphatase 1 & 2.39 \\
\hline mmp10 & Matrix metallopeptidase 10 (stromelysin 2) & 2.06 & $g 6 p c$ & Glucose-6-phosphatase, catalytic subunit & 2.38 \\
\hline s1pr1 & Sphingosine-1-phosphate receptor 1 & 2.03 & pdhal & Pyruvate dehydrogenase (lipoamide) alpha 1 & 2.24 \\
\hline ephb4 & EPH receptor B4 & 1.97 & $c k m$ & Creatine kinase, muscle & 2.24 \\
\hline$n r 2 f 2$ & Nuclear receptor subfamily 2, group F, member 2 & 1.95 & fh & Fumarate hydratase & 2.20 \\
\hline efnb3 & Ephrin-B3 & 1.94 & ogdh & Oxoglutarate hydrogenase (lipoamide) & 2.19 \\
\hline hif3a & Hypoxia inducible factor 3, alpha subunit & 1.92 & gapdh & Glyceraldehyde-3-phosphate dehydrogenase & 2.19 \\
\hline epha7 & EPH receptor A7 & 1.91 & adh5 & Alcohol dehydrogenase 5 (class III) & 2.18 \\
\hline angpt/2 & Angiopoietin-like 2 & 1.90 & $\operatorname{cox} 5 a$ & Cytochrome c oxidase subunit Va & 2.12 \\
\hline nos2 & Nitric oxide synthase 2 , inducible & 1.85 & pgk1 & Phosphoglycerate kinase 1 & 2.02 \\
\hline cdc42ep2 & CDC42 effector protein (Rho GTPase binding) 2 & 1.83 & fads6 & Fatty acid desaturase 6 & 1.99 \\
\hline efna2 & Ephrin-A2 & 1.83 & mdh2 & Malate dehydrogenase 2, NAD (mitochondrial) & 1.97 \\
\hline$n r 2 f 1$ & Nuclear receptor subfamily 2, group F, member 1 & 1.83 & $\operatorname{cox} 6 a 2$ & Cytochrome c oxidase subunit Vla polypeptide 2 & 1.97 \\
\hline jag1 & Jagged 1 & 1.80 & ndufv1 & $\begin{array}{l}\text { NADH dehydrogenase (ubiquinone) flavoprotein } \\
1,51 \mathrm{kDa}\end{array}$ & 1.94 \\
\hline slit2 & Slit homolog 2 (Drosophila) & 1.79 & fabp3 & Fatty acid binding protein 3 , muscle and heart & 1.94 \\
\hline hey1 & Hairy/enhancer-of-split related with YRPW motif 1 & 1.78 & slcad & $\begin{array}{l}\text { Solute carrier family } 2 \text { (facilitated glucose } \\
\text { transporter), member } 2\end{array}$ & 1.92 \\
\hline hiflan & Hypoxia inducible factor 1, alpha subunit inhibitor & 1.73 & atp5h & $\begin{array}{l}\text { ATP synthase, } H+\text { transporting, mitochondrial Fo complex, } \\
\text { subunit } d\end{array}$ & 1.91 \\
\hline foxcl & Forkhead box C1 & 1.68 & uсp3 & Uncoupling protein 3 (mitochondrial ) & 1.88 \\
\hline efnb2 & Ephrin-B2 & 1.63 & cpt2 & Carnitine palmitoyltransferase 2 & 1.86 \\
\hline jag2 & Jagged 2 & 1.54 & ndufb1 & $\begin{array}{l}\text { NADH dehydrogenase (ubiquinone) } 1 \text { beta } \\
\text { subcomplex, 1, 7kDa }\end{array}$ & 1.82 \\
\hline vegfc & Vascular endothelial growth factor $C$ & 1.36 & $c k m+2$ & Creatine kinase, mitochondrial 2 (sarcomeric) & 1.82 \\
\hline$d / 11$ & Delta-like 1 (Drosophila) & -1.25 & mdh1 & Malate dehydrogenase 1, NAD (soluble) & 1.77 \\
\hline
\end{tabular}


Table 3 Selected differentially expressed genes in fast muscle of exercised adult zebrafish that participate in angiogenesis, immune-related processess and metabolism (Continued)

\begin{tabular}{|c|c|c|c|c|c|}
\hline racl & Ras-related C3 botulinum toxin substrate 1 & -1.57 & sdha & Succinate dehydrogenase complex, subunit A, & 1.74 \\
\hline rock2 & $\begin{array}{l}\text { Rho-associated, coiled-coil containing protein } \\
\text { kinase } 2\end{array}$ & -1.61 & $m t-a t p 6$ & ATP synthase F0 subunit 6 & 1.70 \\
\hline notch2 & Notch 2 & -1.89 & $a c a c b$ & Acetyl-CoA carboxylase beta & 1.70 \\
\hline$c d c 42$ & Cell division cycle 42 & -1.97 & ucp2 & Uncoupling protein 2 (mitochondrial) & 1.68 \\
\hline aggfi & $\begin{array}{l}\text { Angiogenic factor with } \mathrm{G} \text { patch and FHA } \\
\text { domains } 1\end{array}$ & -2.10 & atp5o & $\begin{array}{l}\text { ATP synthase, } \mathrm{H}+\text { transporting, mitochondrial F1 } \\
\text { complex, O subunit }\end{array}$ & 1.68 \\
\hline \multicolumn{2}{|c|}{ Immune-related factors } & & enol & Enolase 1, (alpha) & 1,68 \\
\hline traf6 & $\begin{array}{l}\text { TNF receptor-associated factor 6, E3 ubiquitin } \\
\text { protein ligase }\end{array}$ & 10.78 & $\operatorname{cox} 4 i 1$ & Cytochrome c oxidase subunit IV isoform 1 & 1.68 \\
\hline il17D & Interleukin 17D & 6.51 & $\operatorname{cox} 7 a 21$ & $\begin{array}{l}\text { Cytochrome c oxidase subunit Vlla polypeptide } \\
2 \text { like }\end{array}$ & 1.67 \\
\hline ptgs 1 & Prostaglandin-endoperoxide synthase 1 & 5.81 & $\operatorname{atp} 5 f 1$ & $\begin{array}{l}\text { ATP synthase, } \mathrm{H}+\text { transporting, mitochondrial } \\
\text { Fo complex, subunit B1 }\end{array}$ & 1.65 \\
\hline iraklbp 1 & Interleukin-1 receptor-associated kinase 1 BP 1 & 4.98 & nrf1 & Nuclear respiratory factor 1 & 1.62 \\
\hline irf3 & Interferon regulatory factor 3 & 4.60 & $l d h b$ & Lactate dehydrogenase B & 1.60 \\
\hline il29ra & Interleukin 20 receptor, alpha & 3.91 & adipor2 & Adiponectin receptor 2 & 1.56 \\
\hline$i / 12 b$ & Interleukin 12B & 3.68 & $|p|$ & Lipoprotein lipase & -1.51 \\
\hline il11ra & Interleukin 11 receptor, alpha & 3.24 & eifsb4 & $\begin{array}{l}\text { Eukaryotic translation initiation factor } 2 \mathrm{~B}, \\
\text { subunit } 4 \text { delta, } 67 \mathrm{kDa}\end{array}$ & -1.58 \\
\hline ptgr1 & Prostaglandin reductase 1 & 3.21 & gpi & Glucose-6-phosphate isomerase & -1.76 \\
\hline ptgds & Prostaglandin D2 synthase 21kDa (brain) & 3.18 & ndufaf4 & $\begin{array}{l}\text { NADH dehydrogenase (ubiquinone) complex I, } \\
\text { assembly factor } 4\end{array}$ & -1.88 \\
\hline ptgis & Prostaglandin 12 (prostacyclin) synthase & 2.87 & aldoc & Aldolase C, fructose-bisphosphate & -2.03 \\
\hline il13ra2 & Interleukin 13 receptor, alpha 2 & 2.54 & $p k m$ & Pyruvate kinase, muscle & -2.24 \\
\hline$i \mid 20$ & Interleukin 20 & 2.44 & $h k 2$ & Hexokinase 2 & -2.36 \\
\hline tnfrsfig & $\begin{array}{l}\text { Tumor necrosis factor receptor superfamily, } \\
\text { member } 19\end{array}$ & 2.43 & \multicolumn{2}{|c|}{ Protein synthesis and degradation } & \\
\hline$n k r f$ & NFKB repressing factor & 1.77 & $p d k 2$ & Pyruvate dehydrogenase kinase, isozyme 2 & 6.87 \\
\hline il17rd & Interleukin 17 receptor $D$ & 1.74 & fbxo32 & F-box protein 32 & 6.01 \\
\hline mst1 & $\begin{array}{l}\text { Macrophage stimulating } 1 \text { (hepatocyte growth } \\
\text { factor-like) }\end{array}$ & 1.66 & $p d k 1$ & Pyruvate dehydrogenase kinase, isozyme 1 & 2.19 \\
\hline mif & $\begin{array}{l}\text { Macrophage migration inhibitory factor } \\
\text { (glycosylation-inhibiting factor) }\end{array}$ & -1.50 & foxo3 & Forkhead box $\mathrm{O} 3$ & 2.08 \\
\hline ilf3 & Interleukin enhancer binding factor $3,90 \mathrm{kDa}$ & -1.77 & trim63 & Tripartite motif containing 63, E3 ubiquitin protein ligase & 2.02 \\
\hline ptges3 & Prostaglandin E synthase 3 (cytosolic) & -2.29 & rps6ka1 & Ribosomal protein S6 kinase, 90kDa, polypeptide 1 & 1.96 \\
\hline$i \mid 21 r$ & Interleukin 21 receptor & $-2,59$ & eif4e & Eukaryotic translation initiation factor $4 \mathrm{E}$ & $-1,89$ \\
\hline irak4 & Interleukin-1 receptor-associated kinase 4 & $-2,68$ & eif4ebp 1 & $\begin{array}{l}\text { Eukaryotic translation initiation factor } 4 \mathrm{E} \\
\text { binding protein } 1\end{array}$ & $-2,01$ \\
\hline
\end{tabular}

Data are shown as fold change (FC).

Functional categories are indicated in bold.

(Tables 2 and 3), recently shown to be up-regulated in a zebrafish inactivity model [14], is consistent with the up-regulation of the mRNA expression levels of a large number of genes that code for structural and regulatory contractile elements as well as components of the extracellular matrix in fast muscle of exercised zebrafish. Further support for the activation of this pathway in fast muscle of exercised zebrafish can be found in the down- regulation of the expression of pten, a known inhibitor of PI3K/Akt signaling [20]. These observations reinforce the notion that accretion of myofibrillar proteins is an important contributor to muscle growth in fish [21] and strongly suggest that myofibrillogenesis can be stimulated by exercise-induced contractile activity in adult zebrafish. In support of this hypothesis, we recently reported that the increase in protein deposition in the fast muscle of 
Table 4 Significantly over-represented putative canonical pathways in fast muscle of exercised zebrafish

\begin{tabular}{|c|c|c|}
\hline Ingenuity canonical pathways & p-Value & Ratio \\
\hline Integrin Signaling & $3.28 \mathrm{E}-16$ & $94 / 208$ \\
\hline Protein Ubiquitination Pathway & $3.62 \mathrm{E}-12$ & $103 / 268$ \\
\hline Wnt/ß-catenin Signaling & 5.57E-11 & $75 / 175$ \\
\hline mTOR Signaling & $6.06 \mathrm{E}-06$ & $68 / 211$ \\
\hline TGF- $\beta$ Signaling & 7.22E-06 & $36 / 89$ \\
\hline Ephrin B Signaling & $2.46 \mathrm{E}-05$ & $32 / 82$ \\
\hline Actin Cytoskeleton Signaling & $3.04 \mathrm{E}-05$ & $72 / 239$ \\
\hline IGF-1 Signaling & $6.53 \mathrm{E}-05$ & $38 / 105$ \\
\hline Glycolysis & $1.01 \mathrm{E}-04$ & $14 / 41$ \\
\hline VEGF Signaling & $1.03 \mathrm{E}-04$ & $36 / 104$ \\
\hline AMPK Signaling & 4.67E-04 & $46 / 169$ \\
\hline Calcium Signaling & $6.03 \mathrm{E}-04$ & $58 / 213$ \\
\hline Insulin Receptor Signaling & $1.22 \mathrm{E}-03$ & $44 / 142$ \\
\hline FGF Signaling & $1.53 \mathrm{E}-03$ & $31 / 92$ \\
\hline Chemokine Signaling & $1.72 \mathrm{E}-03$ & $26 / 73$ \\
\hline PI3K/AKT Signaling & $1.75 \mathrm{E}-03$ & $41 / 144$ \\
\hline Fatty Acid $\beta$-oxidation & 1.99E-03 & $14 / 45$ \\
\hline Hypoxia Signaling in the Cardiovascular System & 4.35E-03 & $24 / 67$ \\
\hline PDGF Signaling & 4.63E-03 & $27 / 85$ \\
\hline HIF1a Signaling & 9.07E-03 & $33 / 108$ \\
\hline Notch Signaling & $9.60 \mathrm{E}-03$ & $15 / 43$ \\
\hline Angiopoietin Signaling & $3.00 \mathrm{E}-02$ & $21 / 74$ \\
\hline
\end{tabular}

The associated $p$-value (Fisher's exact test $P<0.05$ ) and the ratio of the number of differentially expressed genes in fast muscle of exercised zebrafish over the total number of genes in each particular pathway in the Ingenuity Knowledge Base. Canonical pathway names are from Ingenuity Systems.

swimming rainbow trout [22] was associated with the transcriptional activation of a large set of genes involved in protein biosynthesis and in muscle contraction and development, including components of the sarcomeric structure of skeletal muscle [23]. Interestingly, in the present study exercise also increased the mRNA expression levels of known regulators of atrophy in skeletal muscle, namely the E3 ubiquitin ligases trim63 and fbxo32 [24] and their transcriptional activators foxo3 [25] and traf6 [26] (Table 3), consistent with previous reports indicating that TRIM63 and FBXO32 mRNA expression levels increase in hypertrophied muscles in humans subjected to resistance training [27]. These observations suggest that genes involved in the regulation of the degradation of skeletal muscle protein (i.e. atrogenes), in addition to a large set of genes belonging to the ubiquitin proteasome pathway or other proteolytic systems (e.g. calpains), may also participate in the hypertrophic response of the zebrafish fast muscle to exercise-induced contractile activity, possibly to facilitate the maintenance of normal skeletal muscle protein turnover during long-term training [27]. Therefore, our results strongly indicate that exercise-induced hypertrophy of fast muscle fibres in adult zebrafish involves increased protein turnover, shown for the first time in this species by the parallel activation of the IGF-1/PI3K/ mTOR signaling and atrophy pathways that, in turn, induce the expression of a number of downstream genes coding for myofibrillar elements, as illustrated by the molecular interactome of the muscle development process (Figure 3).

One of the important and novel findings of our transcriptome analysis of the hypertrophic fast muscle of exercised adult zebrafish is the activation of nearly all TGF $\beta$ superfamily signaling pathways known to regulate skeletal muscle mass in mammals. On one hand, we observed an increase in the mRNA levels of follistatin $(f s t)$, known to promote muscle hypertrophy in mammals by binding myostatin (MSTN) and preventing its interaction with activin receptors resulting in activation of the Akt/mTOR signaling pathway to stimulate protein synthesis [28]. The MSTN signaling pathway, known in mammals and fish to exert a repressive action on muscle hypertrophy $[29,30]$ through its inhibition of IGF-1/Akt signaling [31], was also up-regulated in fast muscle of exercised zebrafish as evidenced by the increased expression of the extracellular ligand ( $m s t)$, corroborating the results of our previous study [17], receptors (acvr1b and $a c v r 2 b)$ and signaling molecules (smad2). On the other hand, a number of components of the bone morphogenetic protein (BMP) signaling pathway, including extracellular ligands (bmp1, bmp3, bmp4, bmp $8 b)$, receptors (mbpr1a, bmpr1b), gene targets (id1) and antagonists such as noggin and smad6, were also all up-regulated in fast muscle of exercised zebrafish. In mammals, BMPs promote skeletal muscle hypertrophy by stimulating mTORdependent anabolism [32,33]. The results from the present study are significant because they suggest, for the first time, that the BMP signaling pathway may be involved in exercise-induced hypertrophy of skeletal muscle. In mammals, it has been proposed that the regulation of muscle mass depends on the balance between the competing MSTN and BMP signaling pathways [32]. We hypothesize that the exercise-induced increase in muscle mass associated with hypertrophy of fast muscle in adult zebrafish may have resulted, at least in part, from alterations in the normal balance between negative (MSTN) and positive (FST, BMPs) regulators of skeletal muscle mass.

Importantly, our study also provides molecular evidence to suggest that exercise in adult zebrafish may have activated a myogenic program resulting from the activation of satellite cells. Satellite cells, muscle precursor cells with stem cell characteristics [34], are known to contribute importantly to postnatal skeletal muscle growth and muscle regeneration after injury. However, their involvement in hypertrophic muscle growth in adult mammals is currently 


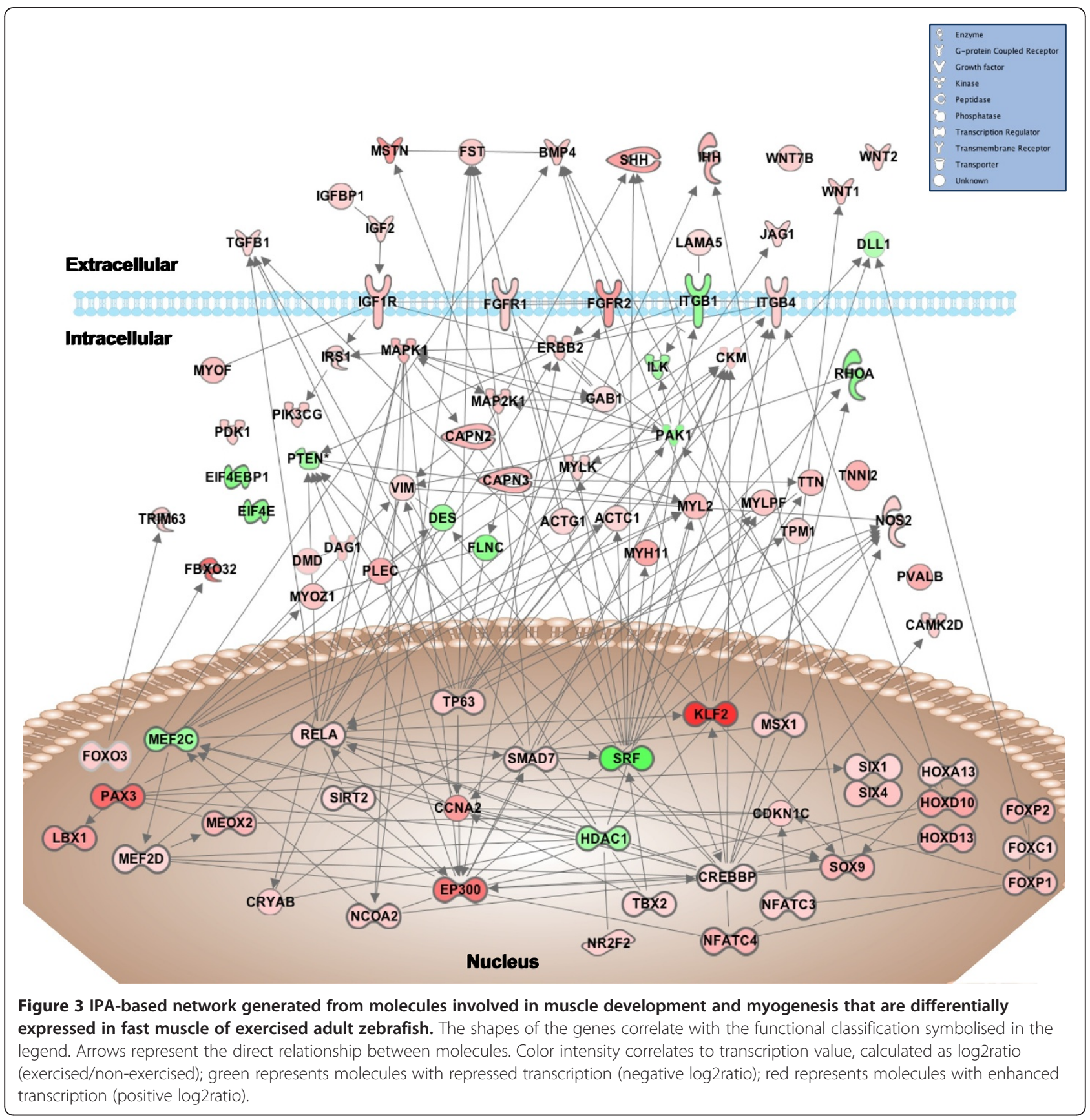

a subject of debate, particularly in the light of studies showing that hypertrophy does not require the presence of satellite cells [35] or their activation [36,37]. In contrast, postembryonic muscle growth in zebrafish is accomplished by mosaic hyperplasia (i.e. new myotubes forming on the surface of existing muscle fibres) until fish achieve half of their final body length after which growth is only accomplished by hypertrophy [21]. To date, the exact role of satellite cells (refered to as myogenic precursor cells in fish) in exercise-induced activity in skeletal muscle or whether contractile activity of skeletal muscle fibres can modify the quiescent status of satellite cells and promote their activation in adult muscle are two aspects that are not completely understood. However, there are reports showing that hypertrophy due to resistance training in humans is associated with an increase in the satellite cell pool probably as a result of increased proliferation [38]. Here, we show for the first time in fish that exerciseinduced activity in adult zebrafish increased the expression of genes known to participate in the myogenic program, most notably the satellite cell marker pax3 and its target gene $l b x 1$. PAX3 is a key factor in skeletal muscle 


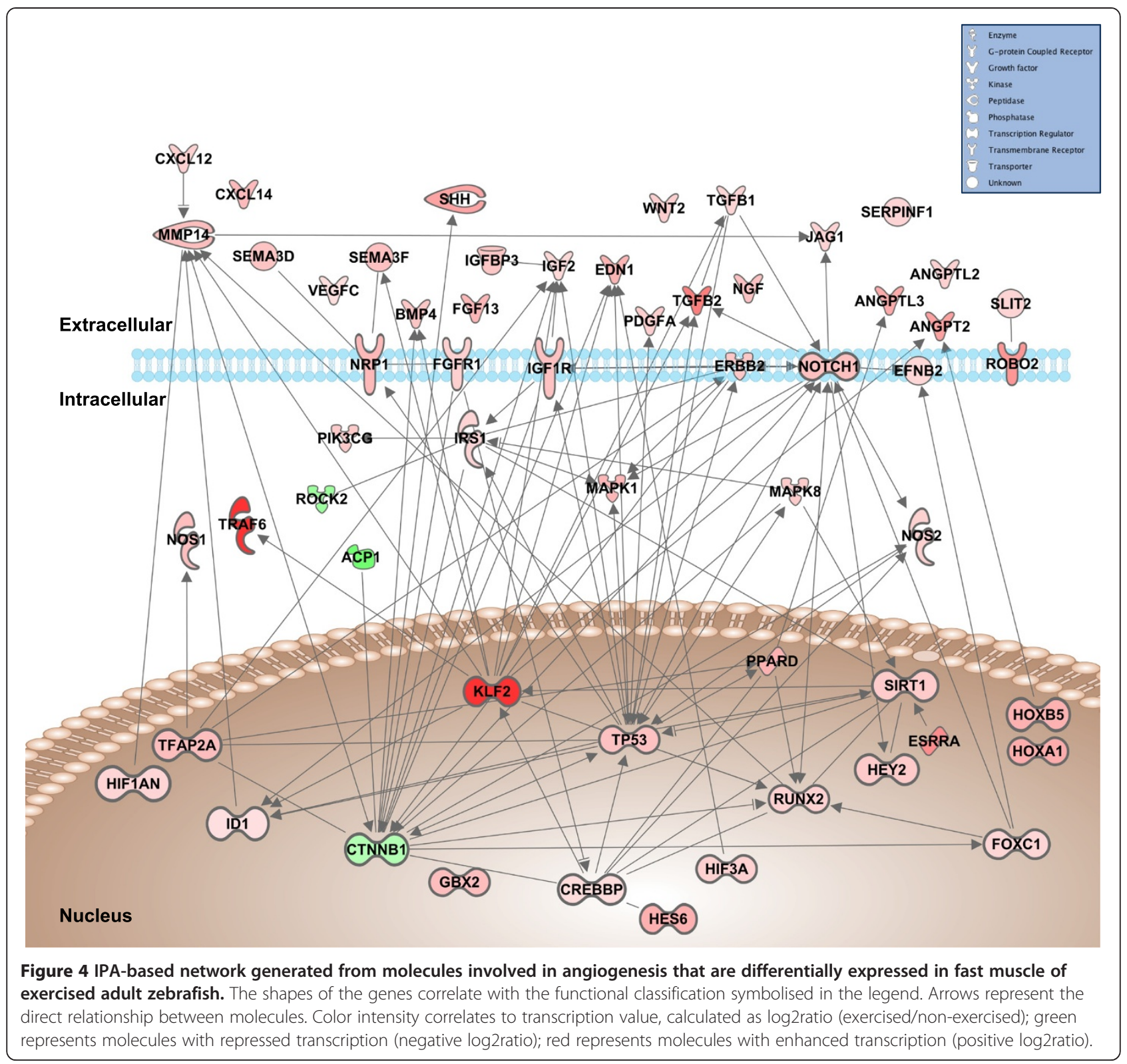

development thought to be responsible for the enlargement of the satellite cell population in muscle at least in part through its activation of the FGF signaling pathway [4]. PAX3 is important for the activation of the muscle regulatory factors MYOD and, together with the mesenchyme homeobox gene 2 (MEOX2) and SIX proteins (SIX1 and SIX4), of MYF5 [4]. PAX3 was recently shown to be up-regulated specifically in hyperplastic growth zones in the late embryonic myotome in rainbow trout [39], another fish species with hyperplastic growth continuing into adulthood. In the present study, we show that the mRNA expression levels of a number of components of the FGF signaling pathway, including ligands $(f g f 13, f g f 18, f g f 20)$, receptors $(f g f r 1, f g f r 2$, $f g f r l 1)$ and signaling molecules (mapk1, raf1, mapk13, crebbp), as well as meox2, six 1 and six4, were increased in fast muscle in response to exercise training in adult zebrafish. All these factors interact with pax3, sox9 and rela in a complex molecular network similar to that described in the exercise-trained human skeletal muscle $[40,41]$. Interestingly, the canonical Notch and Wnt signaling pathways, known to sequentially control the transition of satellite cells from a proliferative to a differentiative phase [42], were also significantly altered in fast muscle of exercised zebrafish. In accordance with the increased expression of pax3, the altered expression of ligands (dll1, jag1, jag2) and receptors (notch1, notch2) of the Notch signaling pathway, coupled with the significant alteration of the 
expression of genes involved in mitosis and cell cycle progression (Additional files 6 and 9: Table S6 and Figure S1), suggests that satellite cells may have been activated by exercise. The recent demonstration that satellite cells in adult zebrafish muscle fibres can be activated by mechanical stretch [43] and that pax3 is expressed in satellite cells isolated from adult zebrafish muscle [44] provide support for the hypothesis that satellite cells may have proliferated in fast muscle of adult zebrafish in response to exerciseinduced activity. In addition, exercise caused a significant increase in the expression of components of the Wnt (e.g. wnt1, wnt2, wnt4, wnt6, wnt7a, wnt7b, wnt8a, wnt10a, wnt10b, wnt11, wnt16; fzd2 to 5, fzd8 to 10; dvl1, dvl2, ccnd1) and the hedgehog (e.g. shh, ihh) signaling pathways, known to play a key role in the induction of myogenesis in vertebrates by promoting differentiation of satellite cells $[8,45]$. Interestingly, hyperplastic growth in embryonic trout was also associated with an important up-regulation of growth factors and soluble signaling molecules (including members of the Wnt pathway) [39] but, to our knowledge, this is the first report of exercise regulating the expression of the hedgehog signaling pathway. However, the expression of various paralogs of fast skeletal myosin heavy chain (e.g. myhz1.1, myhz1.2, myhz1.3 and myhz2) that were reported to be markers for hyperplastic growth in zebrafish [15] did not change in fast muscle of exercised adult zebrafish. Therefore, it will be important to investigate in future studies whether exercise can promote proliferation and/or activation of satellite cells in fast muscle of adult zebrafish.

Exercise-induced activity also altered the mRNA expression levels of other important myogenic differentiation factors in the zebrafish fast muscle, most notably Myocyte enhancer factor 2 (mef2) and serum response factor (srf). MEF2 family members are transcription factors that do not have intrinsic myogenic activity but control the differentiation of skeletal muscle during development through transcriptional cooperation with co-activators such as CREBBP(CBP)/p300, resulting in the potentiation of the function of myogenic regulatory factors (MRFs) and in the regulation of fibre type-specific gene expression programs in mammals [46]. In the adult mammalian muscle, MEF2, in addition to NFAT proteins, is induced by contractile activity in a calcineurin- and CAMKIV-dependent fashion [47] to regulate the metabolic and structural (contractile) phenotype of skeletal muscle cells. Several mef2 genes are expressed in the zebrafish skeletal muscle [48], with mef $2 a$ being expressed in fast muscle after differentiation, mef $2 c$ after myoblast terminal differentiation and mef $2 d$ in muscle precursor cells [49]. Although Mef2c and Mef2d proteins are not required for muscle fibre terminal differentiation, they are indispensable for myofilament expression and myofibril assembly in zebrafish fast muscle fibres [49]. Recently, mef2ca was shown to be induced post-transcriptionally by the TOR pathway to regulate hypertrophic muscle growth in zebrafish [14]. Here, we observed an up-regulation of the mRNA levels of ep300 and crebbp, two nuclear genes that occupy a central position in the transcriptional network in fast muscle of exercised zebrafish (Figure 3), and of mef $2 a$ and $m e f 2 d$; however, the expression of mef2ca was decreased by exercise. In addition, genes involved in calcium signaling initiated by nerve-elicited electrical activity and that regulate MEF2 activity such as ppp3ca (calcineurin), its targets nfatc1, nfatc 3 and nfatc 4, camk4 and hdac4 were all up-regulated by exercise in the zebrafish fast muscle. Another central molecule in the transcriptional network of regulated nuclear genes in the fast muscle of exercised zebrafish is SRF, a transcription factor that regulates myogenic fusion and differentiation and that is also required for overload-induced hypertrophy in the adult mammalian muscle by controlling satellite cell proliferation [50]. The altered expression of $s r f$ in fast muscle of exercised zebrafish, as well as that of the transcriptional repressor $h d a c 1$, is consistent with their role as regulators of skeletal myogenesis [50,51].

\section{Exercise training promotes vascularization in fast muscle of adult zebrafish}

In addition to the increased hypertrophy of fast muscle fibres, exercise increased vascularization of this tissue in adult zebrafish. This is consistent with the well-known increase in capillary number that accompanies fibre hypertrophy in humans and mammalian models $[52,53]$ and also with previous reports that indicate that swim training increases muscle capillarity in several fish species, including larval zebrafish [54-57]. In mammals, exerciseinduced angiogenesis is believed to be induced by the contractile activity of skeletal muscle fibres that, through the combination of growth factor production, hypoxia and shear and mechanical stresses, results in the activation of pro-angiogenic signaling pathways [58]. Importantly, our transcriptomic profiling of the fast muscle of exercised adult zebrafish clearly evidenced the activation of the majority of signaling pathways known in mammals and zebrafish to regulate angiogenesis [59-62], and identified for the first time the molecular programs responsible for the observed increase in vascularization of this tissue by exercise. Specifically, fast skeletal muscle of exercised zebrafish increased the mRNA levels of genes involved in vascular sprouting, including sema3d, sema3f, netrin1 and efnb2, molecules known to be important for intersegmental vessel formation in zebrafish [62], as well as of robo 2 and slit2, an endothelial cell guidance receptor and its ligand, respectively. In addition, exercise also activated at the transcriptional level several canonical signaling pathways known to control the specification of arteries and veins (e.g. Vegf, Notch, Ephrin B2) [63,64], 
as supported by the increased mRNA levels of ssh, of members of the Vegf signaling pathway including ligands (e.g. vegfc), co-receptors (nrp1) and downstream signaling molecules (pik3c2a, pikc3b, pik3cg, plcg1, mapk1), of $n o t c h 1$ and of efnb2 and its receptor ephb4. Furthermore, exercise altered the mRNA levels of genes involved in vascular lumen formation in zebrafish such as integrins, $c d c 42, r a c 1$ and pax2 [62]. Interestingly, to the best of our knowledge, we provide the first demonstration that exercise increases the mRNA levels in fast muscle of $k l f 2$, a shear stress-responsive transcription factor that is activated by the onset of blood flow in newly formed vessels and that induces vessel remodelling through alteration of PI3K and MAPK signaling in zebrafish [65]. klf2 occupies a central position in the angiogenic transcriptional network in fast muscle of exercised adult zebrafish with connections with soluble pro-angiogenic factors (e.g. endothelins, angiopoietins, IGF2, semaphorins), signaling molecules (e.g. traf6, erbb2) and transcriptional regulators (e.g. id1, ctnnb1, crebbp, sirt1) (Figure 4). Remarkably, klf2, as well as other components of the angiogenic transcriptional network such as the IGF-1, TGF $\beta$ and Notch signaling pathways and the nuclear transcriptional regulator crebbp, also participate in the muscle development network (Figure 3). Thus, the molecular response to exercise in skeletal muscle may involve the coordinated activation of angiogenic and muscle development transcriptional programs.

The mechanisms by which angiogenesis is initiated under the normal conditions of adaptive remodelling imposed by exercise are complex and not entirely understood, even in humans. It has been proposed that mechanical and metabolic stimuli responsible for exerciseinduced angiogenesis exert their effects by stimulating the production of VEGF, considered to be a central proangiogenic factor in the regulation of physiological angiogenesis [52,66]. In the present study, we report that exercise-induced contractile activity in adult zebrafish caused changes in the expression of the VEGF canonical pathway and of factors that participate in its regulation including members of the hypoxia-inducible factor family (hif1an, hif3a), nitric oxide synthases (nos 1 and nos2), ppard, known to increase VEGF production and skeletal muscle angiogenesis [67], and esrra, an important mediator of hypoxia-induced PGC-1 $\alpha$ transcriptional regulation of VEGF [68]. Therefore, these results suggest that exercise in adult zebrafish may have induced a transcriptional angiogenic program, at least in part, by activating VEGF and its signaling in fast muscle. In support of this hypothesis, swim training in larval zebrafish was recently reported to increase the expression of the HIF and VEGF pathways [69]. To the best of our knowledge, we provide the first evidence that exercise training in zebrafish activates a complex transcriptional program in fast muscle involving multiple signaling pathways (e.g. VEGF, HIF, TGF $\beta$, Ephrin-B, PDGF, angiopoietin) known to participate in the induction and regulation of angiogenesis, resulting in an important increase in vascularization of this tissue.

We hypothesize that, as in mammals [58], the increase in capillarity as a result of exercise training may enhance the exchange of respiratory gasses, substrates and metabolites between the blood and fast muscle. Consequently, by increasing the oxygen exchange capacity and the ensuing oxidative capacity, exercise may induce a more aerobic phenotype in fast muscle in zebrafish, in agreement with previous studies that showed that swim training increased the aerobic capacity of the fast muscle by increasing the expression of respiratory genes in adult zebrafish [70,71] and in developing zebrafish, as shown by the increased expression of erythropoietin and myoglobin [72]. Support for an increased aerobic phenotype of fast muscle in exercised zebrafish is derived from the observed increased expression of a large set of genes that participate in oxidative metabolism in mitochondria (i.e. TCA cycle and oxidative phosphorylation) and of the oxygen transport protein myoglobin. Although we do not have direct evidence for an effect of exercise on mitochondrial biogenesis, it is interesting to point out that the relationship between capillary and fibre density ( $\mathrm{C} / \mathrm{F}$ ratio), shown here to increase in adult zebrafish in response to exercise as in mammals [58], is related to mitochondrial volume [73] suggesting that swimming-induced exercise could have improved mitochondrial function and number. Surprisingly, the theoretical maximum diffusion distance from the capillaries to the mitochondria increased in fast muscle of exercised zebrafish. Although this finding could initially suggest a reduction in muscle oxidative capacity, it should be only seen as a consequence of fibre hypertrophy. The exerciseinduced increase in capillarization of fast fibres relative to their area and perimeter provides further support for the hypothesis of increased mitochondrial oxidative capacity of fast muscle fibres in adult zebrafish subjected to aerobic exercise training.

\section{Conclusions}

In the present study we have shown that exerciseinduced contractile activity in adult zebrafish promotes a coordinated adaptive response in fast muscle that leads to increased muscle mass by hypertrophy and increased vascularization by angiogenesis. We hypothesize that these phenotypic adaptations are the result of extensive transcriptional changes induced by exercise. Analysis of the transcriptional networks that are activated in response to exercise in the adult zebrafish fast muscle allowed us to identify signaling pathways and transcriptional regulators that play an important role in the regulation of skeletal muscle mass, myogenesis and 
angiogenesis by exercise. The present study is the first to describe coordinated molecular programs regulating muscle mass and vascularization induced by exercise in any species other than humans [74] and supports the notion that these programs may regulate "generic" features of exercise adaptation in the vertebrate skeletal muscle. The development of these adaptive responses to exercise in the zebrafish fast muscle, together with an important metabolic remodelling of this tissue, strongly suggest that exercise training may have caused the acquisition of a more aerobic phenotype in fast muscle in zebrafish. It will be interesting to determine in future studies if these changes result in improved aerobic work capacity. In summary, exerciseinduced activity resulted in the transcriptional activation of a series of complex networks of extracellular and intracellular signaling molecules and pathways involved in the regulation of muscle mass, myogenesis and angiogenesis in adult zebrafish, some of which had not previously been associated with exercise-induced contractile activity. The results from this study demonstrate the utility of the adult zebrafish as an excellent exercise model for advancing our knowledge on the basic mechanisms underlining the regulation of skeletal muscle mass.

\section{Methods}

\section{Ethical approval}

Experiments complied with the current laws of the Netherlands and were approved by the animal experimental committee (DEC number 09161).

\section{Experimental fish and conditions}

Wild-type zebrafish purchased from a local pet shop were housed in two Blazka-type swim tunnels of 127 liters [17] at $28^{\circ} \mathrm{C}$ where approximately 500 liters of fresh water were recirculated over a biofilter system. The photoperiod regime was 16L:8D and they were fed twice a day (DuplaRin pellets, Dupla, Gelsdrof, Germany) before and after each daily training session. In total, two separate experiments were performed: Experiment 1 was described previously [17] and Experiment 2 was executed under the exact same conditions. In each of the two experiments, one swim tunnel contained the non-exercised group (Experiment 1: $\mathrm{n}=83$; Experiment 2: $\mathrm{n}=30$ ) and the other tunnel contained the exercised fish (Experiment 1: $\mathrm{n}=84$; Experiment 2: $\mathrm{n}=30$ ).

\section{Group-wise long term exercise training protocol}

In our previous study [17], a swim training protocol was established for adult zebrafish, where the optimal swimming speed $\left(\mathrm{U}_{\mathrm{opt}}\right)$ was determined at $0.396 \pm 0.019 \mathrm{~m} \mathrm{~s}^{-1}$ or $13.0 \pm 0.6$ standard body lengths $\mathrm{s}^{-1}$. Exercised fish swam at $U_{\text {opt }}$ for 6 hours per day during 20 experimental days while non-exercised fish rested at a lower swimming speed of $0.1 \mathrm{~m} \mathrm{~s}^{-1}$. After 20 experimental days, fish were anesthetised with $1 \mathrm{ml}$ clove oil (10\% in absolute ethanol) in 1 liter of fresh water and euthanized by decapitation. In Experiment 2, exercised fish showed significantly higher body weight than non-exercised fish $(0.34 \pm 0.02 \mathrm{~g}$ vs. $0.25 \pm 0.02 \mathrm{~g}, \mathrm{P}<0.05)$, confirming the results of Experiment 1 [17]. Dorsal epaxial fast muscle filets were dissected and either immediately frozen in isopentane cooled to $-160^{\circ} \mathrm{C}$ and stored in liquid nitrogen until sectioned for histochemical analyses (Experiment 2) or stored at $-20^{\circ} \mathrm{C}$ in RNA later (Life Technologies, Barcelona, Spain) for microarray analyses (Experiment 1 ).

\section{Muscle histochemical analyses}

Fast muscle samples for histochemical analyses were obtained from non-exercised and exercised zebrafish from Experiment 2. After placing the frozen samples in OCT embedding medium at $-22^{\circ} \mathrm{C}$, serial transverse sections of $16 \mu \mathrm{m}$ in thickness were obtained in a cryostat (Leica CM3050S, Wetzlar, Germany) and mounted on 2\% gelatinised slides. Two histochemical assays were performed on fast muscle serial sections: (1) succinate dehydrogenase (SDH) according to [75] in order to demonstrate the aerobic or anaerobic characteristics of muscle fibres; and (2) endothelial ATPase according to [76] to reveal muscle capillaries.

All morphofunctional measurements of fast muscle cellularity and vascularization were performed on the sections processed for endothelial ATPase activity by using a light microscope (BX61, Olympus, Tokyo, Japan) connected to a digital camera (DP70, Olympus). Image Capturing software (DP Controller v. 1.1.1.65, 2002 Olympus) and Image Managing software (DP Manager v. 1.1.1.71, 2002 Olympus) were used to obtain digital microphotographs and to ensure accurate calibration of all measurements. All the parameters listed below were empirically determined from windows of tissue of approximately $5,5 \times \cdot 10^{5} \mu \mathrm{m}^{2}$ from two different zones or muscle fields in each sample using ImageJ analyzing software (v. 1.47, National Institutes of Health, USA). After testing for the absence of differences between the two muscle fields from each sample, the data obtained from both fields were considered together so that the sample size was large enough. The mean results presented throughout tables and figures were obtained from a sample of $\mathrm{n}=8$ fish for each condition (non-exercised and exercised).

In order to determine if swimming-induced exercise caused changes in the morphometric and vascularization characteristics of fast muscle fibres, the following parameters were counted or calculated: capillary density (CD; capillary counts per unit cross-sectional area of muscle), fibre density (FD), capillary-to-fibre ratio $(\mathrm{C} / \mathrm{F}=\mathrm{CD} / \mathrm{FD}$; a parameter relatively independent of FCSA and a good indicator of muscle capillarization [73]), the number of 
capillaries in contact with each fibre (NCF) and the circularity shape factor $\left(\mathrm{SF}=4 \cdot \pi \cdot \mathrm{FCSA} / \mathrm{FPER}^{2}\right)$, which is an estimation of the circular morphology of the fibre (with a value of 1 for a perfect circle). Capillary and fibre counts were calculated and expressed as capillaries and fibres per $\mathrm{mm}^{2}$. The following fibre morphometric parameters were measured: fibre cross-sectional area (FCSA) and perimeter (FPER) and the maximal diffusion distance (MDD) between the capillary and the centre of the fibre. The total number of fibres analyzed in each muscle sample ranged from 200 to 250 . The indices expressing the relationship between the number of capillaries per fibre and the fibre cross-sectional area $\left(\mathrm{CCA}=\mathrm{NCF} \cdot 10^{3} / \mathrm{FCSA}\right)$ or fibre perimeter $\left(\mathrm{CCP}=\mathrm{NCF} \cdot 10^{2} / \mathrm{FPER}\right)$ were also calculated. These indices are considered a measure of the number of capillaries per $1,000 \mu \mathrm{m}^{2}$ of muscle FCSA and the number of capillaries per $100 \mu \mathrm{m}$ of muscle FPER. Data for all the parameters are expressed as sample means \pm standard error of the mean (SEM).

The histograms of FCSA (Figure 1I-K) express the percentage frequencies of fibres grouped in intervals of 200 $\mu \mathrm{m}^{2}$ and error bars represent the SEM. To obtain the superposed curves in the histograms, a dynamic fitting by nonlinear regression was performed for each group of fish (non-exercised and exercised). The approximation was done by a log-normal (four parameters) equation with a dynamic fit option of 200 for both total number of fits and maximum number of iterations. The $R$ values and parameters of the log-normal equations $\left(\mathrm{a}, \mathrm{b}, \mathrm{x}_{0}\right.$ and $\mathrm{y}_{0}$ ), reported with their SEM, are shown in Additional file 1.

\section{Microarray analyses}

Single color microarray-based gene expression analysis was performed using an Agilent custom oligo microarray $4 \times 44 \mathrm{~K}$ with eArray design ID 021626 and containing a total of 43.863 probes of 60 oligonucleotides in length. Total RNA from fast skeletal muscle samples of individual adult zebrafish from Experiment 1 (non-exercised, $\mathrm{n}=8$; exercised, $\mathrm{n}=8$ ) was isolated with TRIzol (Invitrogen, Barcelona, Spain). RNA concentrations of the 16 samples used for microarray analyses, as measured with a NanoDrop ND-1000 (Thermo Scientific), ranged from

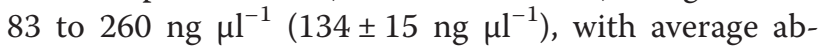
sorbance measures (A260/280) of $2.04 \pm 0,03$, and RNA Integrity Number (RIN) values of $8.85 \pm 0.35$, as obtained using a 2100 Bioanalyzer system (Agilent Technologies, Santa Clara, CA), that were indicative of clean and intact RNA suitable for microarray analysis. RNA was amplified and labeled with $\mathrm{Cy} 3$ dye using single color Low Input Quick Amp Labeling kit (Agilent Technologies) following the manufacturer's indications using $200 \mathrm{ng}$ of RNA in each reaction. Next, $1.65 \mu \mathrm{g}$ of labeled cRNA were hybridized to the arrays. Overnight hybridization (17 h, $65^{\circ} \mathrm{C}$ and $10 \mathrm{rpm}$ rotation) was performed in a Microarray Hybridization Oven (Agilent Technologies). After hybridization, microarrays were washed with Gene Expression Wash Buffers 1 and 2 (Agilent Technologies) and scanned using the High-Resolution C Scanner (Agilent Technologies). Feature Extraction Software 10.7.3 (Agilent Technologies) was used for spot to grid alignment, feature extraction and quantification. Processed data were subsequently imported into GeneSpring GX 11.5 (Agilent Technologies). Significance cut-offs for the ratios of exercised vs non-exercised were set at at $\mathrm{P}<0.01$ (sample t-test) and $>1$-fold change for differentially expressed genes (DEGs). For the DEGs, gene IDs were converted to human ENSEMBL gene IDs using g:orth function from G: profiler (http://biit.cs.ut.ee/gprofiler), taking advantage of the more complete gene ontology (GO) annotations of the human genes and improving, in this way, the subsequent analysis of the functional categories. The complete microarray data have been deposited in NCBI's Gene Expression Omnibus and are accessible through GEO Series accession number GSE58929 (http://www.ncbi.nlm. nih.gov/geo/query/acc.cgi?acc=GSE58929). GO enrichment analysis was performed using Database for Annotation, Visualization and Integrated Discovery (DAVID) software tools (http://david.abcc.ncifcrf.gov), and the resulting categories were considered significant at $\mathrm{P}<0.05$. Pathway and network analyses were conducted using Ingenuity ${ }^{\circ}$ Systems Pathway Analysis (IPA) software (Redwood City, CA). To analyze by IPA, annotated spots were mapped to zebrafish and human orthologs using BLASTN against the Ensembl Danio rerio gene database (v.Zv9.66) and the Homo sapiens transcript database (v.GRCh37.66) with an $e$-value $\leq 1.00 \mathrm{E}-05$. Human and zebrafish orthologs were then compared to the Ingenuity ${ }^{\circ}$ Knowledge Base (www.ingenuity.com) and significantly altered pathways and biological functions were determined using the Fisher exact test $(\mathrm{P}<0.05)$.

\section{Quantitative real-time PCR (qPCR)}

Quantitative real time PCR analysis was performed using RNA treated with RQ1 RNase-free DNase (Promega) to remove any contaminating genomic DNA and reverse transcribed using SuperScript III Reverse Transcriptase (Invitrogen), as specified by the manufacturer. Reactions were run in a MyiQ Real-Time PCR Detection System (Bio-Rad, Madrid, Spain) under the following thermal cycling conditions: $2 \mathrm{~m}$ at $50^{\circ} \mathrm{C}, 8 \mathrm{~min}$ at $95^{\circ} \mathrm{C}$, followed by 40 cycles of $15 \mathrm{~s}$ denaturation at $95^{\circ} \mathrm{C}$ and $30 \mathrm{~s}$ at corresponding melting temperature, and a final melting curve of 81 cycles from $55^{\circ} \mathrm{C}$ to $95^{\circ} \mathrm{C}\left(0.5^{\circ} \mathrm{C}\right.$ increments every $10 \mathrm{~s}$ ) to identify the presence of primer dimers and to analyze the specificity of the reaction. The reactions $(20 \mu \mathrm{l})$ contained $200 \mathrm{nM}$ final concentration of each amplification primer, $10 \mu \mathrm{l}$ of SYBR GreenER qPCR 
SuperMix (Invitrogen) and $5 \mu \mathrm{l}$ of a 1:25 dilution of cDNA for reference gene and target genes. All PCR reactions were run in triplicate (including the nontemplate controls) and fluorescence was measured at the end of each extension step. Efficiency of PCR reactions was calculated by analyzing serial dilutions of pooled cDNA samples and was always higher than $99 \%$. The $2^{-\Delta \Delta C t}$ method [77] was used for real-time PCR analysis and the threshold cycle $(\mathrm{Ct})$ for each gene was normalized to the Ct of RPS15 as reference gene, chosen because of the lack of changes in its expression between exercised and non-exercised zebrafish as assessed by microarray analysis. Primer sequences, amplicon sizes and Ensembl accession numbers of the selected genes are presented in Additional file 10: Table S9.

\section{Statistical analyses}

For capillarization and fibre morphometrical parameters, the normality of the data was tested by the KolmogorovSmirnov test (with Lilliefors' correction) and the comparisons between the two groups of fish (non-exercised and exercised) were analysed by Student's $t$ tests. To test the differences between non-exercised and exercised fish in the frequencies for three intervals of FCSA measured (i.e. fibres with areas below $1.200 \mu \mathrm{m}^{2}$, between 1.200 and $2.400 \mu \mathrm{m}^{2}$ and above $2.400 \mu \mathrm{m}^{2}$; Additional file 1: Table S1), Student's $t$ tests were performed. The normalizing arcsine transformation was applied as a previous step. All statistical analyses were performed using SigmaStat 4.0 (in SigmaPlot 11.0 Software, Systat Software Inc., San Jose, CA, USA).

\section{Additional files}

Additional file 1: Table S1. Equation parameters for the log-normal regression of the fiber cross-sectional area histograms in the fast muscle of zebrafish.

Additional file 2: Table S2. Biological functions that were significantly altered (Fisher's exact test, $\mathrm{p}<0.05$ ) in zebrafish fast muscle in response to swimming.

Additional file 3: Table S3. List of differentially expressed genes involved in the development of muscle in the zebrafish fast muscle in response to exercise.

Additional file 4: Table S4. List of differentially expressed genes involved in myogenesis in the zebrafish fast muscle in response to exercise.

Additional file 5: Table S5. List of differentially expressed genes involved in angiogenesis in the zebrafish fast muscle in response to exercise.

Additional file 6: Table S6. List of differentially expressed genes involved in cell proliferation in the zebrafish fast muscle in response to exercise.

Additional file 7: Table S7. Canonical pathways that were significantly altered (Fisher's exact test, $p<0.05$ ) in zebrafish fast muscle in response to swimming. The number of differentially expressed genes in relation to the total number of genes present in each pathway in the Ingenuity Knowledge Base (No. Genes) and their identity (Pathway molecules) are shown.
Additional file 8: Table S8. Quantitative real-time PCR ( $q P C R$ ) validation of microarray results from selected genes.

Additional file 9: Figure S1. IPA-based network generated from molecules involved in cell proliferation that are differentially expressed in fast muscle of exercised adult zebrafish. The shapes of the genes correlate with the functional classification symbolised in the legend. Arrows represent the direct relationship between molecules. Color intensity correlates to transcription value, calculated as log2ratio (exercised/non-exercised); green represents molecules with repressed transcription (negative log2ratio); red represents molecules with enhanced transcription (positive log2ratio).

Additional file 10: Table S9. Sequences of primers used in gene expression analyses by qPCR.

\section{Competing interests}

The authors declare that they have no competing interests.

\section{Authors' contributions}

Conceived and designed the experiments: APP, MR, JT, HP, JVP. Performed the experiments: APP, MR, DR, JT. Analyzed the data: APP, MR, DR, JT, JVP. Wrote the paper: APP, MR, JVP. All authors read and approved the final manuscript

\section{Acknowledgements}

The authors would like to thank E. Burgerhout, B. Brittijn and C. Tudorache (Leiden University, The Netherlands) for their assistance with Experiment 2; Dr. G. van den Thillart (Leiden University, The Netherlands) for access to two swim-tunnels; G. Goetz and Dr. P. Swanson (National Marine Fisheries Service, NOAA, USA) for their assistance with IPA analyses; Dr. D. Crespo for help with microarray analyses and P. Marquez for her assistance with morphometric analyses. This study was supported by grants from the Ministerio de Ciencia e Innovacion, Spain (CSD2007-0002 and AGL2012-40031-C02-01 to JVP). APP was supported by Marie Curie intra European fellowship FP7-IEF-2007 (REPRO-SWIM; grant agreement number 219971) and a Marie Curie integration grant FP7-PEOPLE-2011-CIG (SWIMFIT; grant agreement number PCIG10-GA-2011303500) from the European Commission. MR was supported in part by a grant from Sudoe-Interreg-EU (AQUAGENET) to JVP.

\section{Author details}

${ }^{1}$ Departament de Fisiologia i Immunologia, Facultat de Biologia, Universitat de Barcelona, Barcelona, Spain. ${ }^{2}$ Institut de Biomedicina de la Universitat de Barcelona (IBUB), Barcelona, Spain. ${ }^{3}$ Institute for Marine Resources and Ecosystem Studies (IMARES), Wageningen Aquaculture, Wageningen UR, Yerseke, The Netherlands. ${ }^{4}$ Department of Molecular Cell Biology, Institute Biology, Leiden University, Leiden, The Netherlands.

Received: 2 July 2014 Accepted: 11 December 2014 Published: 18 December 2014

\section{References}

1. Gundersen K: Excitation-transcription coupling in skeletal muscle: the molecular pathways of exercise. Biol Rev Camb Philos Soc 2011, 86:564-600.

2. Egan B, Zierath JR: Exercise metabolism and the molecular regulation of skeletal muscle adaptation. Cell Metab 2013, 17:162-184.

3. Berchtold MW, Brinkmeier $H$, Muntener M: Calcium ion in skeletal muscle: Its crucial role for muscle function, plasticity, and disease. Physiol Rev 2000, 80:1215-1265

4. Braun T, Gautel M: Transcriptional mechanisms regulating skeletal muscle differentiation, growth and homeostasis. Nat Rev Mol Cell Biol 2011, 12:349-361.

5. Buckingham M, Rigby PWJ: Gene regulatory networksand transcriptional mechanisms that control myogenesis. Dev Cell 2014, 28:225-238.

6. Haskell WL, Lee I-M, Pate RR, Powell KE, Blair SN, Franklin BA, Macera CA, Heath GW, Thompson PD, Bauman A: Physical activity and public health: updated recommendation for adults from the American college of sports medicine and the American heart association. Med Sci Sports Exerc 2007, 39:1423-1434.

7. Colberg SR, Sigal RJ, Fernhall B, Regensteiner JG, Blissmer BJ, Rubin RR, Chasan Taber L, Albright AL, Braun B, American College of Sports Medicine, American Diabetes Association: Exercise and type 2 diabetes: the 
American college of sports medicine and the American diabetes association: joint position statement. Diabetes Care 2010, 33:e147-e167.

8. Ochi H, Westerfield M: Signaling networks that regulate muscle development: lessons from zebrafish. Dev Growth Differ 2007, 49:1-11.

9. Jackson HE, Ingham PW: Control of muscle fibre-type diversity during embryonic development: The zebrafish paradigm. Mech Dev 2013, 130:447-457.

10. Gibbs EM, Horstick EJ, Dowling JJ: Swimming into prominence: the zebrafish as a valuable tool for studying human myopathies and muscular dystrophies. FEBS J 2013, 280:4187-4197.

11. Berger J, Currie PD: Zebrafish models flex their muscles to shed light on muscular dystrophies. Dis Mod Mech 2012, 5:726-732.

12. Dou $Y$, Andersson-Lendahl $M$, Arner $A$ : Structure and function of skeletal muscle in zebrafish early larvae. J Gen Physiol 2008, 131:445-453.

13. Catchen JM, Braasch I, Postlethwait JH: Conserved synteny and the zebrafish genome. Methods Cell Biol 2011, 104:259-285.

14. Yogev O, Williams VC, Hinits Y, Hughes SM: elF4EBP3L acts as a gatekeeper of TORC1 in activity-dependent muscle growth by specifically regulating Mef2ca translational initiation. PLOS Bio/ 2013, 11:e1001679.

15. Johnston IA, Lee H-T, Macqueen DJ, Paranthaman K, Kawashima C, Anwar A, Kinghorn JR, Dalmay T: Embryonic temperature affects muscle fibre recruitment in adult zebrafish: genome-wide changes in gene and microRNA expression associated with the transition from hyperplastic to hypertrophic growth phenotypes. J Exp Biol 2009, 212:1781-1793.

16. Hanai J-I, Cao P, Tanksale P, Imamura S, Koshimizu E, Zhao J, Kishi S, Yamashita M, Phillips PS, Sukhatme VP, Lecker SH: The muscle-specific ubiquitin ligase atrogin-1/MAFbx mediates statin-induced muscle toxicity. J Clin Invest 2007, 117:3940-3951.

17. Palstra AP, Tudorache C, Rovira M, Brittijn SA, Burgerhout E, Van Den Thillart GEEJM, Spaink HP, Planas JV: Establishing zebrafish as a novel exercise model: swimming economy, swimming-enhanced growth and muscle growth marker gene expression. PLoS One 2010, 5:e14483.

18. Schiaffino S, Dyar KA, Ciciliot S, Blaauw B, Sandri M: Mechanisms regulating skeletal muscle growth and atrophy. FEBS J 2013, 280:4294-4314.

19. Davison W, Herbert NA: Swimming-enhanced growth. In Swimming Physiology of Fish. Edited by Palstra AP, Planas JV. Berlin, Heidelberg: Springer-Verlag; 2013:177-202.

20. Small EM, O'Rourke JR, Moresi V, Sutherland LB, McAnally J, Gerard RD, Richardson JA, Olson EN: Regulation of PI3-kinase/Akt signaling by muscle-enriched microRNA-486. Proc Natl Acad Sci U S A 2010, 107:4218-4223.

21. Johnston IA, Bower NI, Macqueen DJ: Growth and the regulation of myotomal muscle mass in teleost fish. J Exp Biol 2011, 214:1617-1628.

22. Felip $\mathrm{O}$, Ibarz A, Fernández-Borràs J, Beltrán M, Martín-Pérez M, Planas JV Blasco J: Tracing metabolic routes of dietary carbohydrate and protein in rainbow trout (Oncorhynchus mykiss) using stable isotopes $\left(\left[{ }^{13} \mathrm{C}\right]\right.$ starch and $\left[{ }^{15} \mathrm{~N}\right]$ protein): effects of gelatinisation of starches and sustained swimming. Br J Nutr 2012, 107:834-844.

23. Magnoni LJ, Crespo D, Ibarz A, Blasco J, Fernández-Borràs J, Planas JV: Comparative biochemistry and physiology, part a. Comp Biochem Physiol A Mol Integr Physiol 2013, 166:1-12.

24. Sandri M: Signaling in muscle atrophy and hypertrophy. Physiology 2008 23:160-170

25. Sandri M, Sandri C, Gilbert A, Skurk C, Calabria E, Picard A, Walsh K, Schiaffino S, Lecker SH, Goldberg AL: Foxo transcription factors induce the atrophy-related ubiquitin ligase atrogin-1 and cause skeletal muscle atrophy. Cell 2004, 117:399-412.

26. Paul PK, Gupta SK, Bhatnagar S, Panguluri SK, Darnay BG, Choi Y, Kumar A: Targeted ablation of TRAF6 inhibits skeletal muscle wasting in mice. J Cell Biol 2010, 191:1395-1411.

27. Leger B, Cartoni R, Praz M, Lamon S, Deriaz O, Crettenand A, Gobelet C, Rohmer P, Konzelmann M, Luthi F, Russell AP: Akt signalling through GSK-3beta, mTOR and Foxo1 is involved in human skeletal muscle hypertrophy and atrophy. J Physiol 2006, 576:923-933.

28. Lee S-J, Lee Y-S, Zimmers TA, Soleimani A, Matzuk MM, Tsuchida K, Cohn RD, Barton ER: Regulation of muscle mass by follistatin and activins. Mol Endocrinol 2010, 24:1998-2008.

29. McPherron AC, Lawler AM, Lee SJ: Regulation of skeletal muscle mass in mice by a new TGF-beta superfamily member. Nature 1997, 387:83-90.

30. Xu C, Wu G, Zohar Y, Du S-J: Analysis of myostatin gene structure, expression and function in zebrafish. J Exp Biol 2003, 206:4067-4079.
31. Trendelenburg AU, Meyer A, Rohner D, Boyle J, Hatakeyama S, Glass DJ: Myostatin reduces Akt/TORC1/p70S6K signaling, inhibiting myoblast differentiation and myotube size. Am J Physiol Cell Physiol 2009, 296:C1258-C1270.

32. Sartori R, Schirwis E, Blaauw B, Bortolanza S, Zhao J, Enzo E, Stantzou A, Mouisel E, Toniolo L, Ferry A, Stricker S, Goldberg AL, Dupont S, Piccolo S, Amthor H, Sandri M: BMP signaling controls muscle mass. Nat Genet 2013, 45:1309-1318.

33. Winbanks CE, Chen JL, Qian H, Liu Y, Bernardo BC, Beyer C, Watt Kl, Thomson RE, Connor T, Turner BJ, McMullen JR, Larsson L, McGee SL, Harrison CA, Gregorevic P: The bone morphogenetic protein axis is a positive regulator of skeletal muscle mass. J Cell Biol 2013, 203:345-357.

34. Wagers AJ, Conboy IM: Cellular and molecular signatures of muscle regeneration: current concepts and controversies in adult myogenesis. Cell 2005, 122:659-667.

35. McCarthy JJ, Mula J, Miyazaki M, Erfani R, Garrison K, Farooqui AB, Srikuea R, Lawson BA, Grimes B, Keller C, Van Zant G, Campbell KS, Esser KA, Dupont-Versteegden EE, Peterson CA: Effective fiber hypertrophy in satellite cell-depleted skeletal muscle. Development 2011, 138:3657-3666.

36. Blaauw B, Canato M, Agatea L, Toniolo L, Mammucari C, Masiero E, Abraham R, Sandri M, Schiaffino S, Reggiani C: Inducible activation of Akt increases skeletal muscle mass and force without satellite cell activation. FASEB J 2009, 23:3896-3905.

37. Lee S-J, Huynh TV, Lee Y-S, Sebald SM, Wilcox-Adelman SA, Iwamori N, Leppe C, Matzuk MM, Fan C-M: Role of satellite cells versus myofibers in muscle hypertrophy induced by inhibition of the myostatin/activin signaling pathway. Proc Natl Acad Sci U S A 2012, 109:E2353-E2360.

38. Petrella JK, Kim JS, Mayhew DL, Cross JM, Bamman MM: Potent myofiber hypertrophy during resistance training in humans is associated with satellite cell-mediated myonuclear addition: a cluster analysis. J App/ Physiol 2008, 104:1736-1742.

39. Rescan P-Y, Montfort J, Fautrel A, Rallière C, Lebret V: Gene expression profiling of the hyperplastic growth zones of the late trout embryo myotome using laser capture microdissection and microarray analysis. BMC Genomics 2013, 14:173.

40. Thalacker-Mercer A, Stec M, Cui X, Cross J, Windham S, Bamman M: Cluster analysis reveals differential transcript profiles associated with resistance training-induced human skeletal muscle hypertrophy. Physiol Genomics 2013, 45:499-507.

41. Keller P, Vollaard NBJ, Gustafsson T, Gallagher IJ, Sundberg CJ, Rankinen T, Britton SL, Bouchard C, Koch LG, Timmons JA: A transcriptional map of the impact of endurance exercise training on skeletal muscle phenotype. J Appl Physiol 2011, 110:46-59.

42. Brack AS, Conboy IM, Conboy MJ, Shen J, Rando TA: A temporal switch from Notch to Wnt signaling in muscle stem cells is necessary for normal adult myogenesis. Cell Stem Cell 2008, 2:50-59

43. Zhang $H$, Anderson JE: Satellite cell activation and populations on single muscle-fiber cultures from adult zebrafish (Danio rerio). J Exp Biol 2014, 217:1910-1917.

44. Alexander MS, Kawahara G, Kho AT, Howell MH, Pusack TJ, Myers JA Montanaro F, Zon LI, Guyon JR, Kunkel LM: Isolation and transcriptome analysis of adult zebrafish cells enriched for skeletal muscle progenitors. Muscle Nerve 2011, 43:741-750.

45. Montarras D, L'honoré $A$, Buckingham M: Lying low but ready for action: the quiescent muscle satellite cell. FEBS J 2013, 280:4036-4050.

46. Potthoff MJ, Olson EN: MEF2: a central regulator of diverse developmental programs. Development 2007, 134:4131-4140.

47. Wu H, Rothermel B, Kanatous S, Rosenberg P, Naya FJ, Shelton JM, Hutcheson KA, DiMaio JM, Olson EN, Bassel-Duby R, Williams RS: Activation of MEF2 by muscle activity is mediated through a calcineurin-dependent pathway. EMBO J 2001, 20:6414-6423.

48. Ticho BS, Stainier DY, Fishman MC, Breitbart RE: Three zebrafish MEF2 genes delineate somitic and cardiac muscle development in wild-type and mutant embryos. Mech Dev 1996, 59:205-218.

49. Hinits $Y$, Hughes SM: Mef2s are required for thick filament formation in nascent muscle fibres. Development 2007, 134:2511-2519.

50. Guerci A, Lahoute C, Hébrard S, Collard L, Graindorge D, Favier M, Cagnard N, Batonnet-Pichon S, Précigout G, Garcia L, Tuil D, Daegelen D, Sotiropoulos A: Srf-dependent paracrine signals produced by myofibers control satellite cell-mediated skeletal muscle hypertrophy. Cell Metab 2012, $15: 25-37$ 
51. Puri PL, lezzi S, Stiegler P, Chen TT, Schiltz RL, Muscat GE, Giordano A, Kedes L, Wang JY, Sartorelli V: Class I histone deacetylases sequentially interact with MyoD and pRb during skeletal myogenesis. Mol Cell 2001, 8:885-897.

52. Egginton S: Invited review: activity-induced angiogenesis. Pflugers Arch 2008, 457:963-977.

53. Plyley MJ, Olmstead BJ, Noble EG: Time course of changes in capillarization in hypertrophied rat plantaris muscle. J Appl Physio/ 1998, 84:902-907.

54. Ibarz A, Felip O, Fernández-Borràs J, Martín-Pérez M, Blasco J, Torrella JR: Sustained swimming improves muscle growth and cellularity in gilthead sea bream. J Comp Physiol B 2010, 181:209-217.

55. Pelster $B$, Sänger AM, Siegele M, Schwerte T: Influence of swim training on cardiac activity, tissue capillarization, and mitochondrial density in muscle tissue of zebrafish larvae. Am J Physiol Regul Integr Comp Physiol 2003, 285:R339-R347.

56. Sänger AM: Effects of training on axial muscle of two cyprinid species: Chondrostoma nasus (L.) and Leuciscus cephalus (L.). J Fish Biol 1992, 40:637-646.

57. Davie PS, Wells RM, Tetens V: Effects of sustained swimming on rainbow trout muscle structure, blood oxygen transport, and lactate dehydrogenase isozymes: evidence for increased aerobic capacity of white muscle. J Exp Zool 1986, 237:159-171.

58. Prior BM: What makes vessels grow with exercise training? J App/ Physiol 2004, 97:1119-1128.

59. Adams $\mathrm{RH}$, Alitalo K: Molecular regulation of angiogenesis and lymphangiogenesis. Nat Rev Mol Cell Biol 2007, 8:464-478.

60. Potente M, Gerhardt H, Carmeliet P: Basic and therapeutic aspects of angiogenesis. Cell 2011, 146:873-887.

61. Chung AS, Ferrara N: Developmental and pathological angiogenesis. Annu Rev Cell Dev Biol 2011, 27:563-584.

62. Gore AV, Monzo K, Cha YR, Pan W, Weinstein BM: Vascular development in the zebrafish. Cold Spring Harb Perspect Med 2012, 2:a006684-a006684.

63. Hong CC, Peterson QP, Hong J-Y, Peterson RT: Artery/vein specification is governed by opposing phosphatidylinositol-3 kinase and MAP kinase/ ERK signaling. Curr Biol 2006, 16:1366-1372.

64. Herbert SP, Huisken J, Kim TN, Feldman ME, Houseman BT, Wang RA, Shokat KM, Stainier DYR: Arterial-venous segregation by selective cell sprouting: an alternative mode of blood vessel formation. Science 2009, 326:294-298.

65. Nicoli S, Standley C, Walker P, Hurlstone A, Fogarty KE, Lawson ND: MicroRNA-mediated integration of haemodynamics and Vegf signalling during angiogenesis. Nature 2010, 464:1196-1200.

66. Hoier $B$, Hellsten $Y$ : Exercise induced capillary growth in human skeletal muscle and the dynamics of VEGF. Microcirculation 2014, 21:301-314

67. Gaudel C, Schwartz C, Giordano C, Abumrad NA, Grimaldi PA: Pharmacological activation of PPARbeta promotes rapid and calcineurin-dependent fiber remodeling and angiogenesis in mouse skeletal muscle. Am J Physiol Endocrinol Metab 2008, 295:E297-E304.

68. Arany Z, Foo S-Y, Ma Y, Ruas JL, Bommi-Reddy A, Girnun G, Cooper M, Laznik D, Chinsomboon J, Rangwala SM, Baek KH, Rosenzweig A, Spiegelman BM: HIF-independent regulation of VEGF and angiogenesis by the transcriptional coactivator PGC-1alpha. Nature 2008, 451:1008-1012.

69. Kopp R, Köblitz L, Egg M, Pelster B: HIF signaling and overall gene expression changes during hypoxia and prolonged exercise differ considerably. Physiol Genomics 2011, 43:506-516.

70. McClelland GB, Craig PM, Dhekney K, Dipardo S: Temperature- and exercise-induced gene expression and metabolic enzyme changes in skeletal muscle of adult zebrafish (Danio rerio). J Physiol 2006, 577:739-751.

71. LeMoine CMR, Craig PM, Dhekney K, Kim JJ, McClelland GB: Temporal and spatial patterns of gene expression in skeletal muscles in response to swim training in adult zebrafish (Danio rerio). J Comp Physiol B 2010 180:151-160.

72. van der Meulen T, Schipper $H$, van den Boogaart JGM, Huising MO, Kranenbarg S, van Leeuwen JL: Endurance exercise differentially stimulates heart and axial muscle development in zebrafish (Danio rerio). Am J Physiol Regul Integr Comp Physiol 2006, 291:R1040-R1048.

73. Mathieu-Costello O, Agey PJ, Wu L, Hang J, Adair TH: Capillary-to-fiber surface ratio in rat fast-twitch hindlimb muscles after chronic electrical stimulation. J Appl Physiol 1996, 80:904-909.
74. Phillips BE, Williams JP, Gustafsson T, Bouchard C, Rankinen T, Knudsen S, Smith K, Timmons JA, Atherton PJ: Molecular Networks of human muscle adaptation to exercise and Age. PLoS Genet 2013, 9:e1003389.

75. Nachlas MM, Tsou KC, De Souza E, Cheng CS, Seligman AM: Cytochemical demonstration of succinic dehydrogenase by the use of a new p-nitrophenyl substituted ditetrazole. J Histochem Cytochem 1957, 5:420-436

76. Fouces V, Torrella JR, Palomeque J, Viscor G: A histochemical ATPase method for the demonstration of the muscle capillary network. J Histochem Cytochem 1993, 41:283-289.

77. Livak KJ, Schmittgen TD: Analysis of relative gene expression data using real-time quantitative PCR and the 2(-Delta Delta C(T)) Method. Methods 2001, 25:402-408.

doi:10.1186/1471-2164-15-1136

Cite this article as: Palstra et al: Swimming-induced exercise promotes hypertrophy and vascularization of fast skeletal muscle fibres and activation of myogenic and angiogenic transcriptional programs in adult zebrafish. BMC Genomics 2014 15:1136.

\section{Submit your next manuscript to BioMed Central and take full advantage of:}

- Convenient online submission

- Thorough peer review

- No space constraints or color figure charges

- Immediate publication on acceptance

- Inclusion in PubMed, CAS, Scopus and Google Scholar

- Research which is freely available for redistribution 